Review

\title{
Femtosecond Laser 3D Fabrication in Porous Glass for Micro- and Nanofluidic Applications
}

\section{Yang Liao and Ya Cheng *}

State Key Laboratory of High Field Laser Physics, Shanghai Institute of Optics and Fine Mechanics, Chinese Academy of Sciences, P.O. Box 800-211, Shanghai 201800, China;

E-Mail: superliao@vip.sina.com

* Author to whom correspondence should be addressed; E-Mail: ya.cheng@siom.ac.cn; Tel.: +86-21-6991-8546; Fax: +86-21-6991-8021.

External Editors: Maria Farsari and Costas Fotakis

Received: 9 October 2014; in revised form: 22 October 2014 / Accepted: 22 October 2014 / Published: 17 November 2014

\begin{abstract}
The creation of complex three-dimensional (3D) fluidic systems composed of hollow micro- and nanostructures embedded in transparent substrates has attracted significant attention from both scientific and applied research communities. However, it is by now still a formidable challenge to build $3 \mathrm{D}$ micro- and nanofluidic structures with arbitrary configurations using conventional planar lithographic fabrication methods. As a direct and maskless fabrication technique, femtosecond laser micromachining provides a straightforward approach for high-precision, spatially-selective, modification inside transparent materials through nonlinear optical absorption. In this paper, we demonstrate rapid fabrication of high-aspect-ratio micro- and/or nanofluidic structures with various 3D configurations by femtosecond laser direct writing in porous glass substrates. Based on this approach, we demonstrate several functional micro- and nanofluidic devices including a 3D passive microfluidic mixer, a capillary electrophoresis (CE) analysis chip, and an integrated micro-nanofluidic system for single DNA analysis. The possible mechanisms behind the formation of high-aspect-ratio micro- and nanochannels are also discussed. This technology offers new opportunities to develop novel 3D micro-nanofluidic systems for a variety of lab-on-a-chip applications.
\end{abstract}

Keywords: femtosecond laser; 3D fabrication; microfluidics; nanofluidics; porous glass 


\section{Introduction}

Microfluidics is a rapidly emerging technology that enables miniaturization and integration for biological, chemical, and medical applications. Integration of fluidic functions such as valving, metering, mixing, transport, and separation on a single substrate has enabled construction of microfluidic systems that can control and manipulate tiny volumes of liquids with high precision, leading to downsizing of both chemical and biological analysis [1,2]. Most current microfluidic systems are primarily two-dimensional. Extension of these devices to three dimensions would allow for full integration of a broad range of different types of component and enable potential high-value applications. However, it is now still a challenge to realize complex three-dimensional (3D) microfluidic structures with current planar photolithography technologies, which require multilayer and multistep processing procedures to form 3D structures, including stacking, bonding, sealing, and so on.

Femtosecond laser direct writing is the main technique currently used to modify the interior of transparent materials in a spatially selective manner [3,4]. This is possible due to the nonlinear interaction between the tightly focused femtosecond laser beam and the transparent material since the interaction is effectively confined to the vicinity of focal point where the laser intensity exceeds the threshold for multiphoton absorption. For instance, femtosecond laser pulses have been used to write optical waveguides in both passive and active materials by locally modifying their refractive indices [5-7]. In combination with wet chemical etching, femtosecond laser direct writing has also been used to fabricate microfluidic structures, including microchannels and chambers [8,9], microvalves [10], and micropumps [11]. The same technique has been extended to fabricate free-space optics such as micromirrors and micro-optical lenses in glass materials [12-14]. By virtue of its unique ability to build different types of functional components into a monolithic glass substrate, femtosecond laser direct writing offers a flexible approach to fabricate a wide variety of integrated microsystems and devices such as microfluidic lasers [15], optofluidic sensors [16-21], micro-electrodes [22-26] and micro-mechanics [27,28].

Currently, there are mainly two strategies for fabricating 3D microfluidic channels embedded in glasses using a femtosecond laser. The first strategy employs femtosecond laser modification inside glass materials followed by chemical wet etching [29-31]. Unfortunately, with this technique the length of the microfluidic channel that can be fabricated is usually limited to a few millimeters, due to the limited etch ratio between the areas with and without the femtosecond irradiation. Another strategy is to perform femtosecond laser 3D drilling from the rear surface of the glass in contact with distilled water, in which the water introduced into the microchannel can help to remove the ablated debris [32]. However, when the length of the channel increases, the debris can no longer be ejected from the microchannel, which in turn causes clogging and termination of the microchannel. So far, the microchannels fabricated by these methods often suffer from their insufficient lengths for practical microfluidic applications.

Another challenge of fabricating 3D fluidic structures by femtosecond laser direct writing is the limited fabrication resolution mainly caused by the optical diffraction limit. So far, by inducing two-photon polymerization (2PP) of photosensitive materials with femtosecond laser pulses, 3D fabrication with a resolution of sub-100 $\mathrm{nm}$ has already been achieved by choosing laser intensity slightly above a threshold value [33]. However, the 2PP technology is intrinsically limited to polymer materials, in which direct formation of thin microfluidic channels cannot be easily achieved due to the difficulty in removing the non-photopolymerized materials contained in the thin channels. Currently, 
the thinnest microfluidic channels were obtained by liquid-assisted femtosecond laser 3D drilling, resulting in channel diameters ranging from 500 to $700 \mathrm{~nm}$ [34].

This review article focuses primarily on our recent efforts to tackle the two issues as mentioned above. By use of femtosecond laser direct writing in porous glass immersed in water followed by post-annealing, we demonstrated microfluidic channels with nearly unlimited lengths and arbitrary 3D geometries $[35,36]$. By controlling the laser peak intensity and polarization, a single nanocrack with sub-50-nm feature size could be achieved inside porous glass [37]. Based on these strategies, several functional devices and their applications are demonstrated, including large-volume hollow chamber [38], 3D passive microfluidic mixer [39], capillary electrophoresis (CE) analysis chip [40], and an integrated micro-nanofluidic system for single DNA analysis [41]. The possible mechanisms behind the formation of a single nanochannel are also discussed [42,43]. Finally, future opportunities and current challenges for this novel approach are highlighted.

\section{Experimental Setup and Fabrication Process}

In this work, a high-silica porous glass was used as a substrate, which was produced by removing the borate phase from phase-separated alkali-borosilicate glass in hot acid solution [44]. The composition of the porous glass is approximately $95.5 \mathrm{SiO}_{2}-4 \mathrm{~B}_{2} \mathrm{O}_{3}-0.5 \mathrm{Na}_{2} \mathrm{O}$ (wt \%). The pores with a mean size of $\sim 10 \mathrm{~nm}$ are distributed uniformly in the glass and occupy $40 \%$ volume of the glass. Particularly, these pores in the porous glass form a $3 \mathrm{D}$ connective network, which allows liquid to flow through.

Figure 1 schematically shows a typical experimental setup used for femtosecond laser direct writing in porous glass, which consists of a femtosecond laser amplifier system (Coherent, Inc., Santa Clara, CA, USA, center wavelength: $\sim 800 \mathrm{~nm}$, pulse width: $\sim 50 \mathrm{fs}$, repetition rate: $1 \mathrm{kHz} \sim 250 \mathrm{kHz}$ ), a Charge-coupled Device (CCD) imaging system, a computer-controlled XYZ translation stage, beam control devices and delivery optics. The Gaussian laser beam with an initial $8.8 \mathrm{~mm}$ diameter was passed through a $\sim 3 \mathrm{~mm}$-diameter aperture for ensuring a high beam quality (i.e., only the central part of the beam was used because of its high homogeneity). A long-working-distance water-immersed objective was employed for focusing the beam into the porous glass sample, which was fixed in a petri dish filled with distilled water. The petri dish was placed on the translation stage, as shown in Figure 1. Since the high-repetition-rate laser pulses could also lead to temporary evacuation of water in the laser action zone by semi-continuous irradiation, a chopper was used to modulate the femtosecond laser pulse train during the ablation process, so that the water can always fill the ablation zone by infiltration through the porous network.

Figure 1. Schematic of the setup used for femtosecond laser direct writing in porous glass.

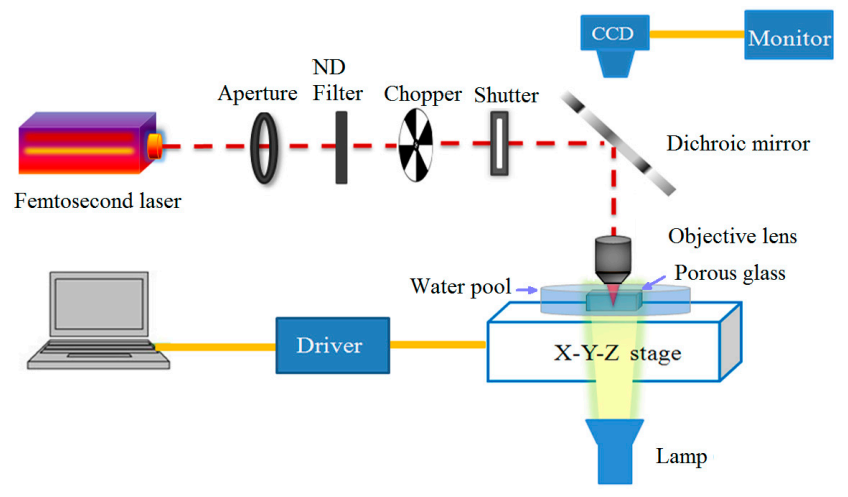


Figure 2a,b shows the schematic view of the experimental setup and the flow diagram for the fabrication process, respectively. The main fabrication process includes two steps: (1) direct formation of $3 \mathrm{D}$ microchannels from one side of a porous glass substrate immersed in water by femtosecond laser direct writing and (2) postannealing of the glass substrate at $\sim 1150{ }^{\circ} \mathrm{C}$ by which the porous glass can be consolidated. The inset scanning electron microscope (SEM) image in Figure 2a clearly shows the size distributions of nanopores are homogeneous. After the consolidation process, all the nanopores in the porous glass disappeared; however, the fabricated microchannels could survive due to their large diameter. Moreover, the porous structure is consolidated into a clear impervious glass known as Vycor brand $96 \% \mathrm{SiO}_{2}$ glass, which is similar to fused silica in its thermal properties and light transmittance [44].

Figure 2. (a) Schematic view of experimental setup. (b) Flow diagram for the fabrication process.

(a)

(b)
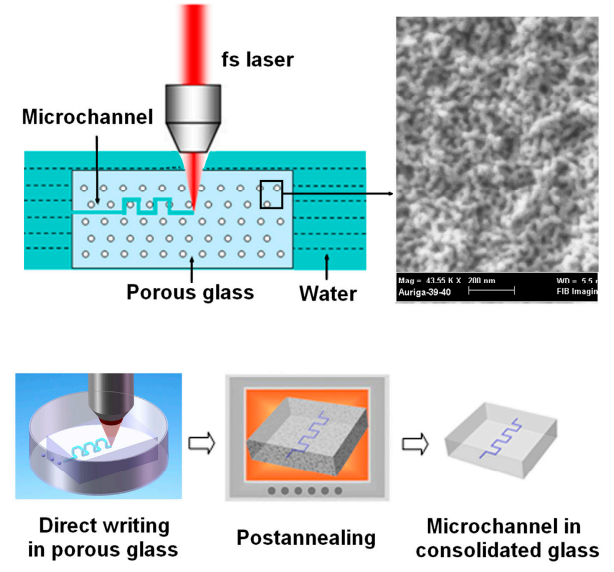

\section{Laser Direct Writing of Microchannels}

\subsection{Fabrication of Multi-Centimeter-Long Microchannels}

For laser direct writing of microchannels in porous glass, relatively high laser intensity is required for inducing laser ablation in microchannel. Typically, a $50 \times$ objective (numerical aperture (N.A.) $=0.80$ ) was employed for focusing the $1 \mathrm{kHz}$ femtosecond laser pulses at a depth of $300 \mu \mathrm{m}$ below the sample surface. The laser pulse energy was chosen to be $60 \mu \mathrm{J}$, and the translating speed was set to be $20 \mu \mathrm{m} / \mathrm{s}$. Figure 3a shows a section of microchannel inside porous glass immersed in water, wherein the dark segments consisted of gas bubbles produced when focusing femtosecond laser pulses on the silica/water interface. The discontinuous bubble segments indicate that some parts of the channel were blocked by debris created by laser ablation. By repeatedly scanning, the water could disperse and flush away clogged segments, until a homogeneous and continuous hollow channel was obtained. The continuous bubble section shown in Figure $3 \mathrm{~b}$ indicates that the channel is homogeneous and debris-free, after one slow scan at $20 \mu \mathrm{m} / \mathrm{s}$ followed by repeatedly scanning the whole microfluidic channel six times at a higher translation speed of $1 \mathrm{~mm} / \mathrm{s}$.

Figure 4a shows a top view micrograph of the homogeneous square-wave-like microchannel embedded in porous glass with a total length of $\sim 1.6 \mathrm{~cm}$, which was obtained by multiple scans with a total machining time of $\sim 15 \mathrm{~min}$. However, the microfluidic channel embedded in porous glass cannot be directly used for microfluidic application, because the liquid in the channel tends to permeate into the surrounding pores. To collapse all the pores, the sample was further annealed at $1150{ }^{\circ} \mathrm{C}$ for $1 \mathrm{~h}$. 
Figure 4b,c shows optical micrographs of the postannealed microchannel filled with red ink. It is clear that the ink is well confined in the microchannel, which indicates that the microchannel is completely sealed because the pores have been closed after postannealing.

It should be specifically mentioned that after the consolidation process, the glass substrate shrinks in all the three directions. The initial length of $\sim 1.6 \mathrm{~cm}$ of the microfluidic channel decreases to $\sim 1.4 \mathrm{~cm}$ after the postannealing. Furthermore, we compare the cross sections of microchannels before and after the postannealing by breaking up the microfluidic channels, as shown in Figure 5a,b. Table 1 gives geometric sizes of microchannel before and after annealing, which indicate the shrinkage factors in three directions are almost equal. The corresponding volume shrinkage factor is $\sim 39 \%$, approximately equal to the $40 \mathrm{vol} \%$ porosity of porous glass. Note that there are some cracks along the laser propagating direction around the microchannels as shown in Figure 5a,b, which could result from inhomogeneous axial intensity distribution of the incident laser due to both self-focusing effect and spherical aberration effect [45].

Figure 3. Optical micrographs of microchannels in porous glass fabricated by (a) single scan and (b) multiple scans.

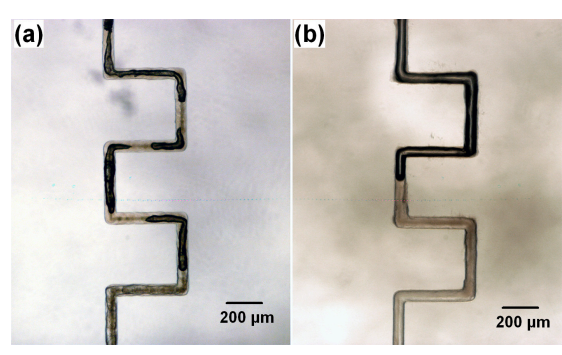

Figure 4. (a) Optical micrograph of a 1.6-cm-long microchannel embedded in porous glass before postannealing. (b) Optical micrograph of postannealed microchannel filled with red ink. (c) Close-up view of (b) [35] (Reproduced with permission from OSA. C2010 by the Optical Society of America).

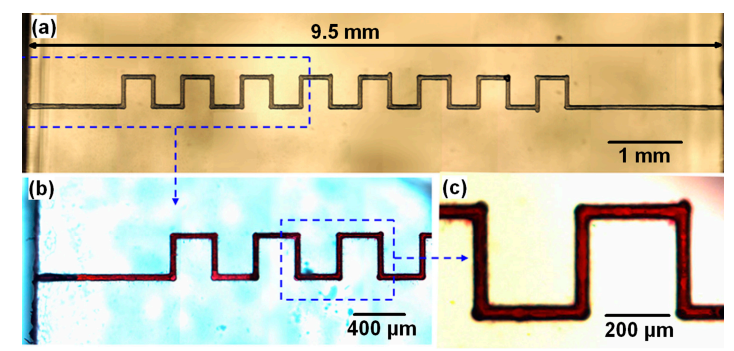

Figure 5. Cross-sectional views of the cleaved microchannel (a) before and (b) after the postannealing [35] (Reproduced with permission from OSA. (C2010 by the Optical Society of America).

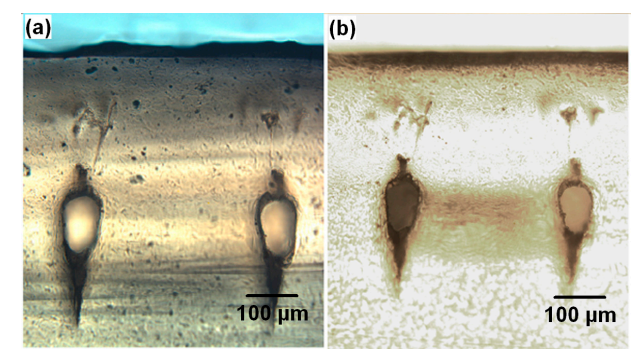


Table 1. Geometric sizes of microchannel before and after annealing.

\begin{tabular}{cccc}
\hline Description & Cross section size (transverse) & Cross section size (vertical) & Channel length \\
\hline Before annealing & $74 \mu \mathrm{m}$ & $114 \mu \mathrm{m}$ & $9.50 \mathrm{~mm}$ \\
After annealing & $63 \mu \mathrm{m}$ & $97 \mu \mathrm{m}$ & $8.00 \mathrm{~mm}$ \\
Shrinkage factor & $14.9 \%$ & $14.9 \%$ & $15.8 \%$ \\
\hline
\end{tabular}

Moreover, the porous glass substrate, which is opaque due to the scattering of the nanopores, becomes highly transparent after the consolidation process, as shown in Figure 6a. Figure $6 \mathrm{~b}$ gives a transmission spectrum of the consolidated glass, which shows superior transmission ranging from ultraviolet radiation (UV) to the near infrared radiation (IR). This facilitates the future incorporation of optical functions in the glass for optofluidic applications.

Figure 6. (a) The porous glass before (left) and after (right) postannealing. (b) Transmission spectrum of the consolidated glass.

(a)

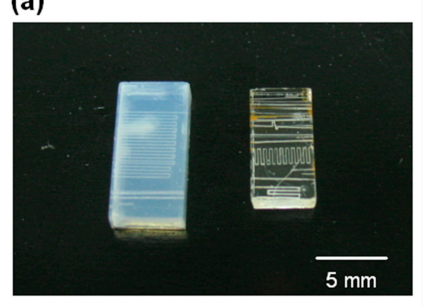

(b)

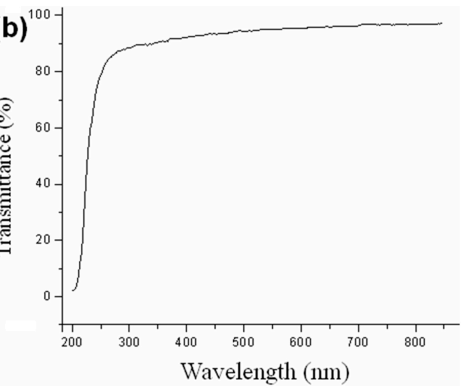

\subsection{Mechanism of Efficient Removal of Debris}

In this section, we discuss the dominant mechanism that is responsible for the removal of debris from the microchannel during the femtosecond laser direct writing in porous glass. Actually, in the previous studies of fabrication of microfluidic channel in fused silica by water-assisted femtosecond laser drilling [32,46], it has been observed that the drilling process relies heavily on water circulation in the microchannel, which is a bi-directional mass transfer process including both the water flowing into laser ablation zone from the inlet of mcirochannel driven by capillary force and bubbles ejecting through the inlet driven by the shock wave produced by the femtosecond laser, as schematically shown in Figure 7a. The water mainly plays two key roles for producing hollow structures: (1) dispersing the debris produced by laser ablation; and (2) producing bubbles to expel the debris from the channel through the inlet, which in return promotes the water circulation. Unfortunately, as the length of channel increases in solid glass, once the bubble and debris build up to the extent that water inflow is blocked, the drilling process will terminate. However, for porous glass, the water can continuously infiltrate into the ablation zone through the porous network, as schematically shown in Figure $7 \mathrm{~b}$, even if the microchannel is blocked. Therefore, the repeated back and forth writing can always disperse debris and produce bubbles independent of the length of microchannel.

In the right panels of Figure $7 \mathrm{a}, \mathrm{b}$, the resistance forces of bubble ejection when microchannel is blocked by bubbles are illustrated for solid glass case and porous glass case, respectively. For porous glass case, it will be much easier to drive the bubbles out from the channel because the capillary force exerted by water surrounding bubbles is balanced out. In our experiment of femtosecond laser direct 
writing in porous glass, somewhere the microchannel could be temporarily blocked if some of the bubbles in the channel do not have enough time to escape from the channel before the new debris is produced. However, we can easily expel the bubbles from the channel by scanning the femtosecond laser beam along the channel repeatedly.

In addition, due to the porosity of the sample, the amount of debris produced by the femtosecond laser ablation would be less than that in the traditional water-assisted drilling inside dense glass. Furthermore, the porosity of the sample will also allow for directly creation of hollow space by localized solidification and the collapse of the nanopores, which has been verified by the formation of hollow nanogratings in porous glass [37].

Figure 7. Schematic illustration of water-assisted direct-writing process in (a) solid glass and (b) porous glass.

(a)
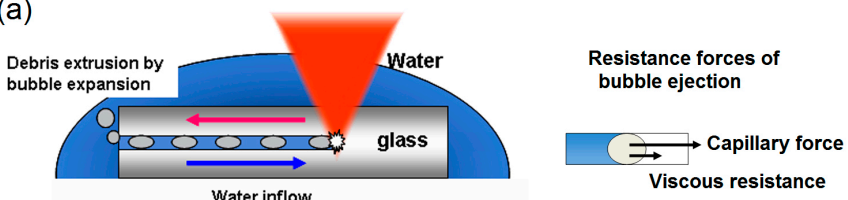

(b)
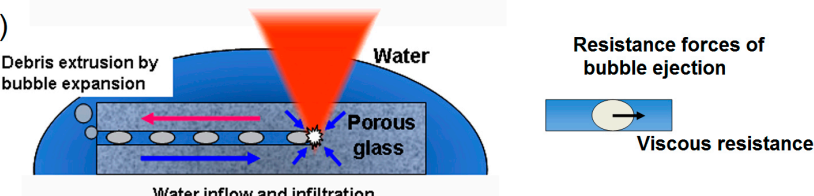

\subsection{High-Aspect-Ratio 3D Microchannels}

For direct writing of high-aspect-ratio (length-to-diameter) microchannels, both high-repetition-rate femtosecond laser pulses and tightly focusing are desirable. Here, the $250-\mathrm{kHz}$ femtosecond laser pulses with a pulse energy of $\sim 2 \mu \mathrm{J}$ were tightly focused by a water-immersed objective (N.A. $=1.0$ ). Under such conditions, a peak laser intensity of $\sim 8 \times 10^{15} \mathrm{~W} / \mathrm{cm}^{2}$ can be reached in a tiny focal volume with a diameter of $\sim 0.8 \mu \mathrm{m}$, which is sufficiently strong for inducing nonlinear optical absorption and ablation of the glass material. The significant amount of heat and strong shock waves generated by the femtosecond laser ablation can facilitate water circulation and help to remove the debris from the outlet of the microchannel. Since the high-repetition-rate laser pulses could also lead to temporary evacuation of water in the laser action zone by semi-continuous irradiation, a chopper operated at $1 \mathrm{kHz}$ with a duty of 50/50 was used (see Figure 1) to modulate the femtosecond laser pulse train during the ablation process, so that the water can always fill the ablation zone by infiltration through the mesoporous network [47].

The 3D nature of the femtosecond laser direct writing offers unique flexibility for constructing complex microfluidic networks [48]. Figure 8a shows a 3D helical microchannel with a total length of $\sim 1 \mathrm{~cm}$ and a diameter of $\sim 16 \mu \mathrm{m}$ (i.e., corresponding to an aspect ratio of $>600$ ). The coil radius and the pitch of the helical channel are $100 \mu \mathrm{m}$ and $50 \mu \mathrm{m}$, respectively. The homogeneous and debris-free helical channel was produced inside porous glass by performing one slow scan $(10 \mu \mathrm{m} / \mathrm{s})$ followed by three fast scans $(100 \mu \mathrm{m} / \mathrm{s})$ over the whole microfluidic channel. The total fabrication time is less than 30 min. Figure $8 \mathrm{~b}$ shows several overpass structures consisted of spiral-like bends along microfluidic 
channels, by which one microchannel can cross over the others. The fluorescence micrograph presented in the inset of Figure $8 \mathrm{~b}$ indicates that indeed, the two microchannels do not intersect each other. The insets in Figure 8a,b presents fluorescence microscopy images of the fabricated microchannels filled with fluorescein solutions. The confined fluorescent solution gives proof that nanopores have all collapsed to form the consolidated substrate.

Many microfluidic applications such as electrophoresis require long channels compactly deployed in a tiny space. Compact microfluidic systems not only reduce the sizes of micro-analysis systems but also promote device performances such as sensitivity. Toward this goal, we fabricated a 3D two-layer microfluidic channel structure in porous glass with a length up to $\sim 6.6 \mathrm{~cm}$. Such a channel length can be sufficient for electrophoresis-based chemical analysis [49,50]. Figure 9a,b shows a microchannel with an aspect ratio of $\sim 5500$ fabricated in porous glass and consolidated glass, respectively. The total length of the microchannel is $6.6 \mathrm{~cm}$ and its diameter is $\sim 12 \mu \mathrm{m}$, and the distance between the two layers was $\sim 30 \mu \mathrm{m}$. In this case, femtosecond laser pulses with a pulse energy of $\sim 3.5 \mu \mathrm{J}$ were tightly focused into porous glass substrate using a water-immersed objective (N.A. $=1.0$ ). The debris-free microchannel was written back and forth at a low translation speed, and the total fabrication time is $\sim 3 \mathrm{~h}$. To our knowledge, this is so far the longest microfluidic channel with the highest aspect ratio produced in a glass substrate by femtosecond laser direct writing. In fact, there is no limit on the channel length with this technique if one would spend enough time on fabrication of the structure.

Figure 8. Optical micrographs of (a) a 3D helical microchannel and (b) 3D microfluidic channels crossing over each other embedded in consolidated glass, Insets: Fluorescence microscopy image of the microchannel filled with fluorescein solutions [39] (Reproduced with permission from RSC. (C)2012 by the Royal Society of Chemistry).

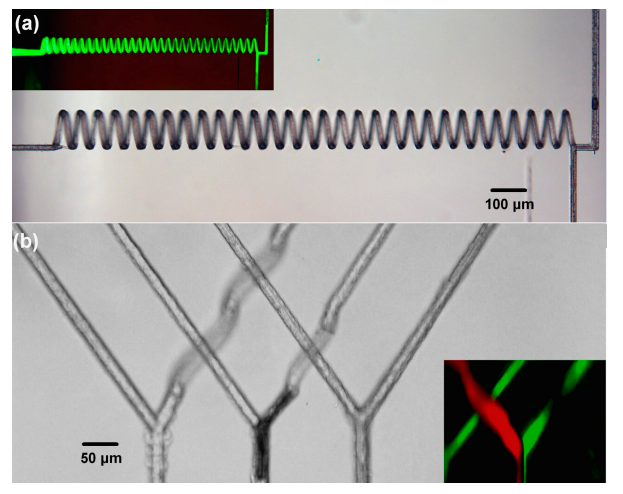

Figure 9. (a) Optical micrograph of a two-layer microfluidic channel in porous glass with a length up to $6.6 \mathrm{~cm}$. (b) Fluorescence microscopy image of the postannealed microchannel filled with a solution of fluorescein.

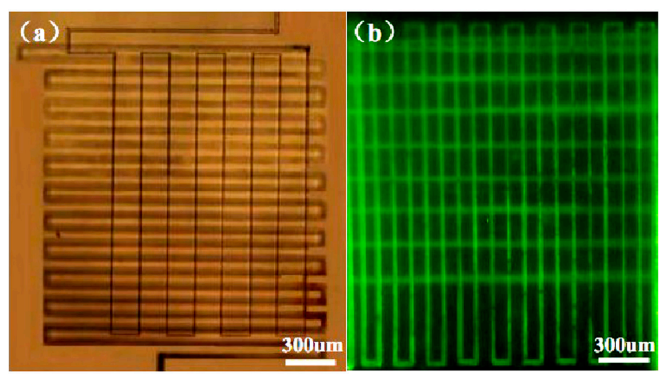




\subsection{Microchannels Fabricated by Nanosecond Laser}

Despite the rapid development of ultrafast laser technology in recent years, femtosecond laser systems are still expensive and difficult to operate as compared to other types of lasers, hampering their applications in industrial environment. In this section, we show that 3D microchannels can also be fabricated in porous glass using a Q-switched, frequency-doubled Nd:YAG laser [51]. The processing mainly consists of two steps: (1) formation of hollow microfluidic channels in porous glass immersed in Rhodamine 6G (Rh6G) dissolved in water by nanosecond laser ablation; and (2) postannealing of the fabricated porous glass sample at $1120{ }^{\circ} \mathrm{C}$ for consolidation of the sample. Here, Rh6G plays an important role as an absorber for promoting the ablation by the nanosecond laser within the porous glass sample, e.g., near the focal volume. The mechanism behind this technique is somewhat similar to that of laser induced backside-wet etching (LIBWE) [52-54], in which glass surface in contact with organic solution can be ablated by nanosecond laser to form surface microstructures. Due to the fact that the porous glass is immersed in a solution doped with Rh6G, the liquid can penetrate into the substrate through the nanopores. Since we use a $532 \mathrm{~nm}$ laser whose wavelength is close to the absorption peak of Rh6G $(\sim 520 \mathrm{~nm})$, we choose the Rh6G in water for achieving an efficient absorption at the focus. Therefore, ablation inside glass is possible. Although the nanosecond laser could result in relatively low fabrication resolution due to its associated heat effect, there is no doubt that replacing the femtosecond laser by the nanosecond laser will greatly reduce the cost for fabricating $3 \mathrm{D}$ microfluidic devices whereas in the meantime enhance the stability of the laser source.

Figure 10a shows a schematic illustration of the system for carrying out nanosecond laser direct writing in porous glass immersed in Rh6G diluted with water at a concentration of $\mathrm{C}=6.0 \times 10^{-3} \mathrm{~mol} / \mathrm{L}$. Before being immersed in the liquid, the porous glass appears opaque due to the strong scattering of light from the nanopores; however, after soaked in the liquid, because of the smaller refractive-index difference between the glass $(n=1.46)$ and the absorbing liquid $(n=1.33)$ as compared to the refractive-index difference between the glass and air $(n=1)$, the scattering of light can be significantly reduced, which facilitates formation of high-quality focal spot inside glass. The laser was operated at a wavelength of $532 \mathrm{~nm}$ with $10 \mathrm{~ns}$ pulse duration (Full width at half maximum (FWHM)) and a repetition rate of $10 \mathrm{~Hz}$. The incident pulse energy was chosen to be $\sim 100 \mu \mathrm{J}$. The nanosecond laser beam was focused into the porous glass through an objective lens (N.A. $=0.8$ ). Some straight microfluidic channels were fabricated inside the porous sample at different depth as illustrated in Figure 10b.

Figure 10. (a) Schematic view of experimental setup and (b) flow chart for the whole fabrication process [51] (Reproduced with permission from OSA. C2012 by the Optical Society of America).

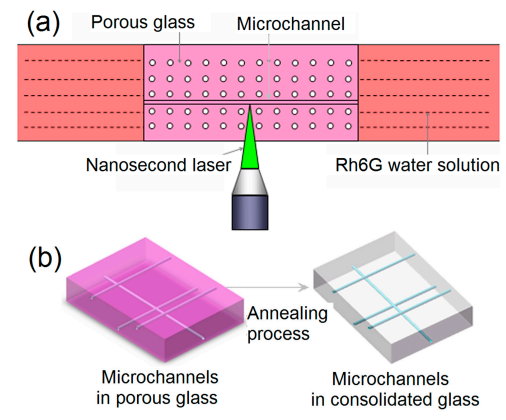


The microchannels were fabricated by a single scan at a translation speed of $10 \mu \mathrm{m} / \mathrm{s}$ until the desired length is achieved, followed by several back-and-forth scans at a translation speed of $100 \mu \mathrm{m} / \mathrm{s}$ for completely removing the debris remaining in the channel. Uniform channels of a total length up to $\sim 1 \mathrm{~cm}$ were achieved after the multiple scan. The total process for fabricating the 1-cm-long channel took $\sim 1 \mathrm{~h}$, limited by the low repetition rate of our YAG laser. After the laser direct writing, a post-annealing process was applied to the sample for collapsing all the nanopores in the glass, so that leak-free microfluidic channels are formed. The temperature inside the furnace was ramped up to $1120{ }^{\circ} \mathrm{C}$ at a rate of $1{ }^{\circ} \mathrm{C} / \mathrm{min}$ and held at $1120^{\circ} \mathrm{C}$ for $2 \mathrm{~h}$ and then naturally cooled down to room temperature. Before the post-annealing, the sample was soaked in acetone to take an ultrasonic bath for 30 min for removing the Rh6G molecules. A small amount of residual Rh6G dye left behind by the ultrasonic bath was further completely removed during the postannealing.

Figure 11a shows a top view optical micrograph of a machined microfluidic channel embedded in porous glass before post-annealing. The left section of the channel was empty and thus appeared dark, while the right section was filled with liquid and then appeared bright. In order to examine the morphology of the innerwall of microchannel, we polished the sample from the top surface until the microchannel was exposed. A SEM image of the surface morphology of the postannealed sample is shown in Figure 11b. It is clear that the innerwalls of the channel after the postannealing process show relatively high surface roughness, which can be attributed to the debris redeposited onto the inner wall of the microchannel. The debris possibly could be reduced or even totally removed by an additional chemical etching in diluted hydrofluoric acid (HF).

Figure 11. (a) Top view optical micrograph of a machined channel embedded in porous glass before postannealing. (b) SEM image of the innerwall of the channel after postannealing.
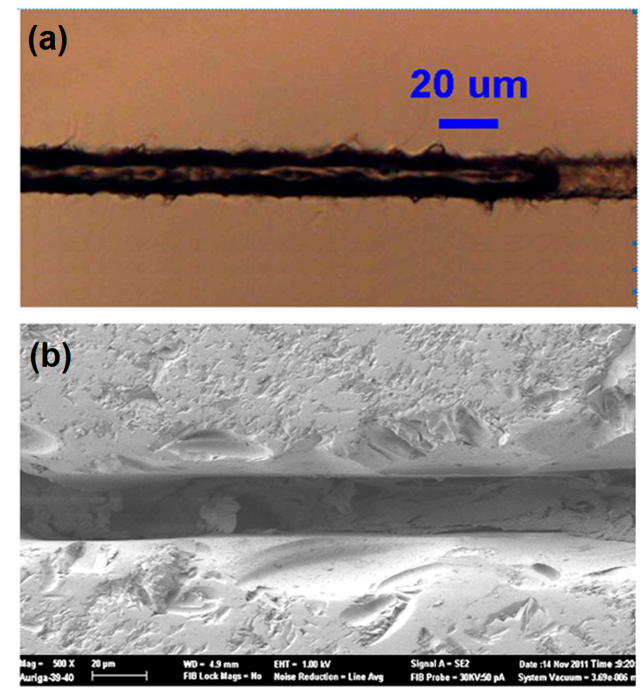

\section{3D Microfluidic Devices}

\subsection{Large-Volume Hollow Chamber}

Recently, 3D cell culture system has attracted wide attention of researchers range from engineering tissues for clinical delivery through to the development of models for drug screening, where both gene expression and other biological activities more closely mirror what happen in living organisms [55]. 
To build 3D microfluidic devices for cell culture, hollow microfluidic chamber with relatively large volume and arbitrary 3D configurations are desirable.

Figure 12 shows the fabrication process of a large-volume chamber inside glass by femtosecond laser direct wiriting. Two straight channels were first fabricated inside the porous sample for inflow of water and ejection of debris. Along with the straight channels, a chamber was fabricated by translating the sample in a line-by-line and layer-by-layer manner. The scan of the chamber started from its bottom in order to avoid the extra aberration created by the previously formed hollow layers. After the laser direct writing, a post-annealing process was applied to the sample for removing all the nanopores in the glass. After postannealing, geometric sizes of the annealed hollow chamber decrease to $85 \%$ of the original one, while its shapes remain unchanged (also see Table 1).

Figure 13a shows a large-volume chamber connected to four microchannels embedded in the porous glass. Here, the $250-\mathrm{kHz}$ femtosecond laser pulses with a pulse energy of $\sim 6.5 \mu \mathrm{J}$ were tightly focused by an objective lens (N.A. $=0.90)$. Firstly, the microchannels were fabricated with one slow scan at a translation speed of $20 \mu \mathrm{m} / \mathrm{s}$. The diameter of the microchannels is approximately $20 \mu \mathrm{m}$. Secondly, the microchamber was fabricated in a line-by-line and layer-by-layer manner at a higher translation speed of $100 \mu \mathrm{m} / \mathrm{s}$. The interval between the adjacent lines in the horizontal plane is $3 \mu \mathrm{m}$, and the vertical interval between the adjunct layers is $5 \mu \mathrm{m}$. This size of the microchamber was $1 \mathrm{~mm} \times 1 \mathrm{~mm} \times 100 \mu \mathrm{m}$ defined by the number of adjacent lines, and the total scanning process for fabricating the chamber took $\sim 20 \mathrm{~h}$ because of its large volume. The thick black curve in Figure 13b shows the boundary of air and water in the chamber, providing a clear evidence of the hollow structure. The technique allows for fabrication of arbitrary 3D hollow structures embedded in glass.

Figure 12. Schematic illustration of the fabrication process for large-volume hollow chamber.

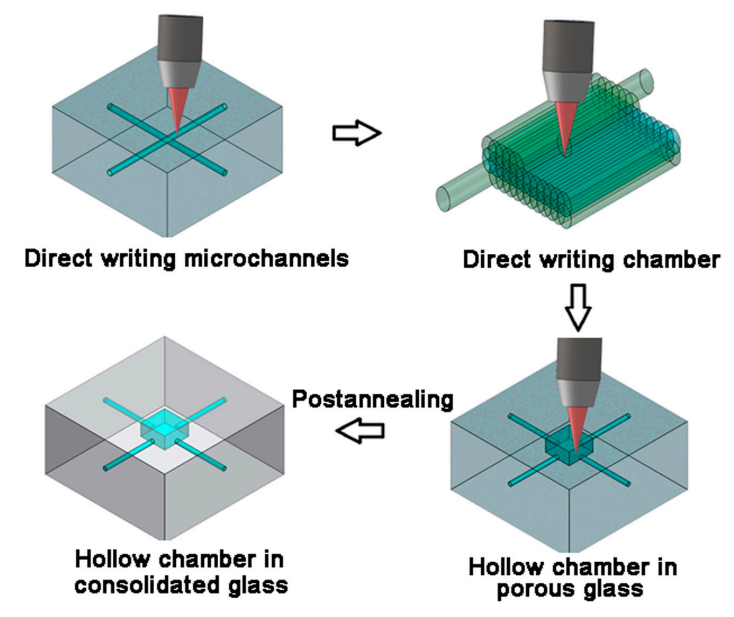

Figure 13. (a) Over view image of a $1 \mathrm{~mm} \times 1 \mathrm{~mm} \times 100 \mu \mathrm{m}$ chamber connected to four microchannels. (b) Enlarged view of the large volume chamber.

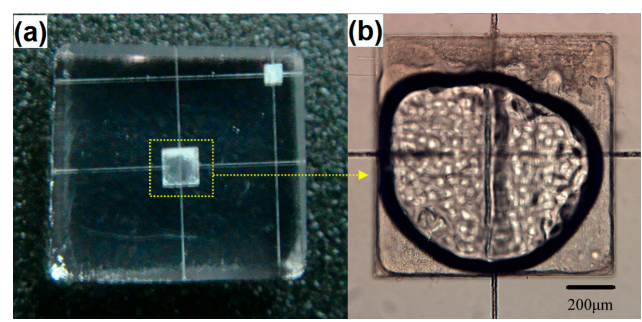




\subsection{Three-Dimensional Microfluidic Mixer}

Fluid mixing is an essential function required by most microfluidic systems. However, fast and efficient fluid mixing inside microchannels is usually difficult to achieve due to the laminar nature of microflows, characterized by low Reynolds numbers. Recently, various passive mixers have been developed to achieve efficient mixing by utilizing 3D geometric structures to induce disturbance in the fluids [56-64]. Nevertheless, the fabrication of 3D microfluidic mixer with arbitrary geometries is still challenging. Here, we demonstrate rapid fabrication of a passive microfluidic mixer consisting of geometrically complex 3D microchannels by femtosecond laser direct writing in porous glass.

According to the chaotic theories, a passive micromixer based on the baker's transformation concept could provide ideal mixing performance [65]. However, such kinds of mixers usually require complicated 3D geometries, thus they are difficult to fabricate by the conventional planar fabrication process. By use of the femtosecond laser direct writing, we fabricated a passive micromixer consisting of symmetrical 3D units as theoretically proposed by Carrière [66]. Figure 14a,b presents the schematic illustrations of the designed 3D mixer which is composed of a Y-shape microchannel embedded $400 \mu \mathrm{m}$ below the surface of chip and a string of mixing units, connected to two opening inlets and one outlet. The inlets and outlet are all on the backside of the chip. Three circular slots surrounding the inlets and the outlet were also fabricated by femtosecond laser ablation, which were used as connection interfaces with plastic pipes of a diameter of $0.9 \mathrm{~mm}$. Figure $14 \mathrm{c}$ presents an overview of a 3D micromixer consisted of six mixing units. The length of all the horizontal and vertical channels in each mixing unit is $150 \mu \mathrm{m}$, as shown in Figure 14d (top view), e (side view). It also can be observed from Figure 14e that the cross section of channel is elliptical with a width of $\sim 50 \mu \mathrm{m}$ and a depth of $\sim 75 \mu \mathrm{m}$. Figure 14f shows the inner surface of the microchannel for evaluation of the surface roughness.

Figure 14. Schematic diagrams of the 3D passive microfluidic mixer: (a) overview and (b) close-up view images. (c) Overview optical micrograph of the microfluidic mixer, (d) top view optical micrograph of two mixing units, and (e) cross sectional optical micrograph of the 3D microchannels. (f) Scanning electronic micrograph of the inner wall of the fabricated microchannel. The dark sections in (e) are caused by the bubbles [39] (Reproduced with permission from RSC. (C2012 by the Royal Society of Chemistry).

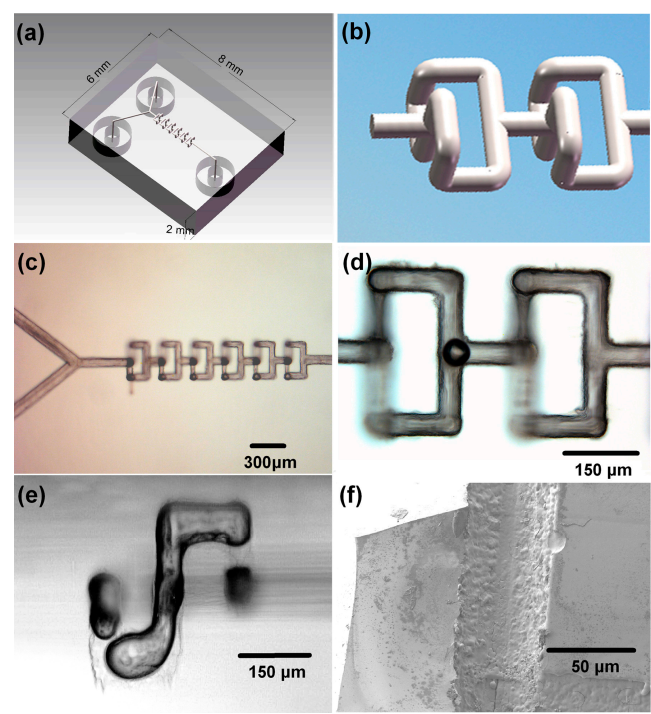


Figure 15a,b shows the experimental results of mixing of the two fluorescent dye solutions (fluorescein sodium and Rhodamine B) in the 1D and 3D microfluidic mixers fabricated under the same direct writing conditions, respectively. Clearly, the mixing behaviors in these two structures agree well with the simultion results. For the 3D mixer, after passing through three mixing units (i.e., a length of $0.9 \mathrm{~mm}$ ), the two fluids are well mixed, corresponding to a mixing time of $\sim 10 \mathrm{~ms}$. For comparison, Figure 15a shows that in the 1D microfluidic channel, efficient mixing was not achieved after a propagation distance of $\sim 1300 \mu \mathrm{m}$. The insets in Figure 15a,b shows the numerical simulations of mixing in 1D and 3D microfluidic mixers, respectively. It can be clearly seen that the experimental results and the computational results are in good agreement.

Figure 15. Fluorescence microscopy images of the (a) 1D and (b) 3D microfluidic mixing experiments, where two fluids (red and green) are mixed at Reynolds number $(\mathrm{Re})=5.3$. Scale bars $=150 \mu \mathrm{m}$. Insets: Numerical simulations of mixing in (a) $1 \mathrm{D}$ and (b) $3 \mathrm{D}$ microfluidic mixers. $\mathrm{Re}=5.3$.

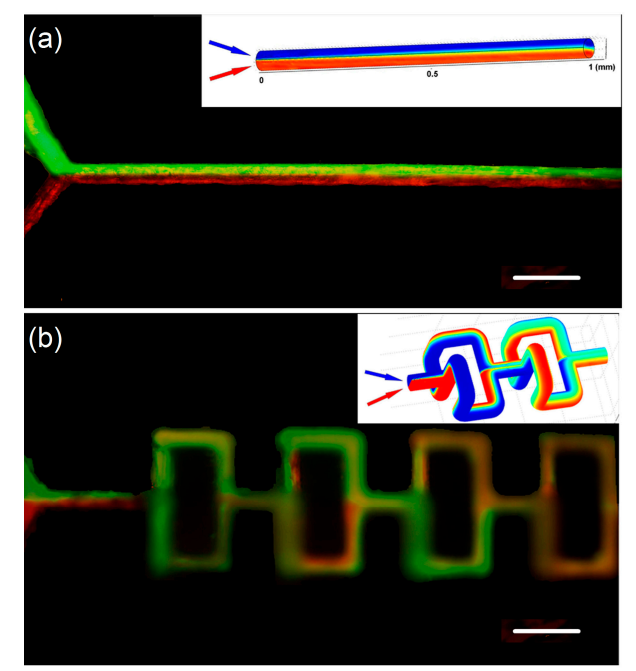

\subsection{Electrophoresis Microchip}

Electrophoresis is an important approach for performing chemical and biological analysis, which achieves separation of charged analytes with different size to charge ratios by accelerating them to different velocities in a conductive liquid in thin capillaries under the influence of an electric field. Nowadays, it is more and more popular to perform CE experiments in microfluidic channels fabricated on microchips, as the microfluidic CE chips typically consume only picoliters of samples and produce less waste [1]. Further development of microfluidic-chip $\mathrm{CE}$ technique requires integration of preparation and analysis functions into the microchips. Toward this goal, femtosecond laser 3D micromachining holds great promise as it has been demonstrated for creating a variety of functional components including microfluidics, microoptics, microelectrodes, etc. [67]. For example, integration of optical waveguides into a commercially produced CE device has been demonstrated by femtosecond laser direct writing, showing enhanced functionality and ease of operation [68]. Nevertheless, in this example, the $\mathrm{CE}$ device is prefabricated using traditional lithography technology, due to the difficulty in forming homogeneous large-scale microfluidic network embedded inside glass with traditional femtosecond laser micromachining techniques. In this Section, we show that the difficulty can be 
solved by direct writing with tightly focused femtosecond laser pulses in the porous glass. We demonstrate a fully functional capillary electrophoresis (CE) analysis chip and test its functionality by observing the motion of three amino acids, namely, L-Histidine, L-Lysine and DL-Alanine in the microfluidic channels.

The completed microchip is shown in Figure 16a, and a close-up view of the corner area of the microchannel is shown in Figure 16b. The smooth edge of the channel indicates that the ablation debris generated during the laser writing has been efficiently removed. The lengths of the microchannel connecting the buffer inlet and outlet and of that connecting the sample inlet and outlet are $35 \mathrm{~mm}$ and $8 \mathrm{~mm}$, respectively, as shown in Figure 16a. In addition, the channels have a depth of $\sim 10 \mu \mathrm{m}$ and a width of $\sim 36 \mu \mathrm{m}$. The channel width could be controlled by scanning the femtosecond laser line-by-line, as we have demonstrated before [38]. We tested the functionality of the CE device fabricated by femtosecond laser by observing the motion of three amino acids, namely, L-Histidine, L-Lysine and DL-Alanine in the microfluidic channels. After the derivatized amino acids were added into the inlet, a voltage of $200 \mathrm{~V}$ was applied across the sampling channel. The voltage applied across the migration channel was $100 \mathrm{~V}$. After $2 \mathrm{~s}$, the amino acids were driven into the junction of the two microchannels. With a migration voltage of $620 \mathrm{~V}$ applied across the migration channel and $200 \mathrm{~V}$ across the sampling channel, movement of the amino acids with a migration velocity of $\sim 0.49 \mathrm{~mm} / \mathrm{s}$ was observed under a fluorescence microscope.

Currently, most CE microchips used to separate analytes are fabricated on polymer/glass substrates based on lithography approaches. Polymer CE chips are easy to fabricate and the inner wall of the microchannel can be easily modified. However, the electro-osmotic flow can be unstable in polymer channels, which will decrease the separation efficiency. In addition, for CE chips fabricated using planar lithography techniques, sealing of the open channels fabricated on the upper substrate surfaces always poses a problem because complete sealing of tiny channels on the micrometer or even nanometer scales frequently causes clogging and/or leakage. We stress that these problems can be well addressed via our technique, as we have shown above.

Figure 16. (a) Overview optical micrograph of the fabricated electrophoresis microchip.

(b) Close-up view of the turning area of the microchannel.

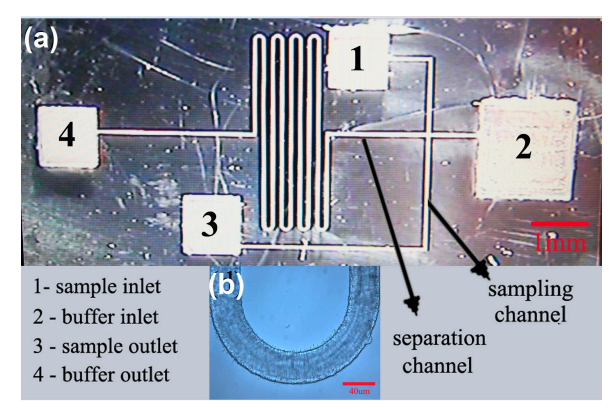

\section{3D Nanofluidic Channels and Devices}

\subsection{Direct Writing of Nanochannels}

Recently, nanofluidics has attracted increasing attention due to its unique fluid transport properties and great potential to handle and probe individual biomolecules [69]. The incorporation of nanofluidic elements into microfluidic devices enables further downsizing of micro-analysis systems and 
enhancement of their functionalities, both of which are highly desired for lab-on-a-chip applications [70]. Both fundamental investigation and practical application of nanofluidics heavily rely on fast and simple nanofabrication techniques [71-73]. Here, we show that by uniquely employing the porous glass as the substrate material, nanogratings consisting of an array of elliptical-shaped hollow nano-voids elongated in the direction of laser propagation can be formed in glass using linearly polarized writing beam. By reducing the peak intensity of the laser pulses close to a threshold value, only a single hollow nano-void in the central area of the focal spot can survive, serving as the basic element for construction of 3D nanofluidics inside glass.

Previously, it has been reported that nanogratings can be formed in glass with femtosecond laser irradiation, which consist of alternating regions of high and low etch rates periodically distributed in a direction perpendicular to the laser polarization [74-76]. We found that the nanograting-like structures can also be induced in the porous glass by femtosecond laser irradiation, whereas the gratings are composed of an array of hollow nano-voids [37], as schematically shown in Figure 17.

Figure 17. Schematic diagram of inducing nanograting structures inside water-immersed porous glass by femtosecond laser pulses. The sample is translated in the $x-y$ plane, the writing polarization is aligned to the $x$-axis, and the laser incident direction is along the $z$-axis [37] (Reproduced with permission from OSA. C2013 by the Optical Society of America).

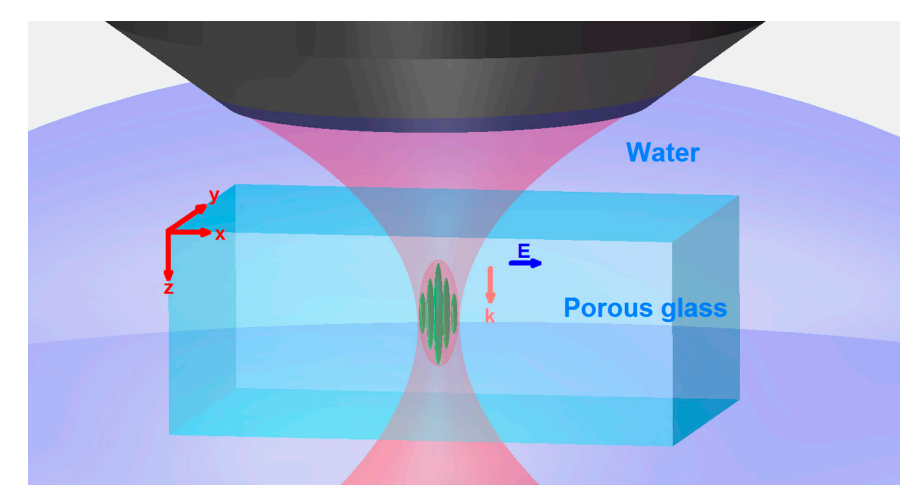

Amazingly, we found that a single nanochannel could still be induced in the central area of the focal volume at very low laser intensity, while other nanograting-like channels vanish. The cross sections of the nanogratings induced by femtosecond laser direct writing at different pulse energies (with a same writing speed of $10 \mu \mathrm{m} / \mathrm{s}$ ) are shown in Figure $18 \mathrm{a}-\mathrm{c}$. The direction of laser polarization is perpendicular to the translating direction. At a relatively high pulse energy of $90 \mathrm{~nJ}$, the number of the elliptical nano-voids in the grating is large, as shown in Figure 18a. By reducing the pulse energy to $70 \mathrm{~nJ}$, the number of the nano-voids decreases to only four. It is noteworthy that under this condition, the periodicity of the nanograting apparently increases as evidenced in Figure 18b, which also helps reduce the number of nano-voids. Finally, by reducing the pulse energy to $60 \mathrm{~nJ}$, only the central nano-void survives, showing an extremely narrow width of $\sim 37 \mathrm{~nm}$ as presented in Figure 18c.

Figure $18 \mathrm{~d}-\mathrm{f}$ presents a principle schematic diagram to achieve a single nanocrack by combining the threshold effect and the formation of periodic nanograting. Conventionally, the surface nanostructuring of glass beyond the diffraction limit could be achieved by taking advantage of a threshold effect, i.e., by bringing the laser intensity down to a level approaching a threshold laser intensity only above which the ablation of glass induced by multiphoton absorption occurs [77,78]. In theory, there is no 
limit on the fabrication resolution when the threshold effect takes place, because the ablation linewidth can always be reduced if one keeps on reducing the difference between the chosen laser intensity and the threshold intensity. However, in practice, due to the fluctuation of the output power of the femtosecond laser, the fabrication process will become extremely unstable when the laser intensity is near the threshold intensity [79]. As a matter of fact, reliable sub-100 nm fabrication linewidth is extremely difficult to achieve for surface ablation. Moreover, sub-100 nm fabrication linewidth has never been demonstrated for 3D nanostructuring in bulk transparent materials so far due to the different physical mechanism involved in bulk machining [80]. In our case, the formation of a single nanocrack is much less sensitive to the fluctuation of the laser intensity by combining the threshold effect and the formation of a periodic nanograting. As shown in Figure 18f, when the femtosecond laser intensity is intentionally reduced to a level at which only the intensity in the blue region is higher than the threshold intensity, one will be able to select only one nanocrack in the central area of the focal volume. Note that the single nanocrack (green) is much narrower than the above-threshold-intensityregion (blue). So there exists a window (a range of laser intensity) for inducing a single nanocrack, which is between the threshold laser intensity of emergence of a single nanocrack and the minimum laser intensity for inducing two nanocracks in porous glass.

The induced single nano-voids can be connected into a continuous structure for construction of 3D nanochannels in glass. This is achieved by slowly scanning the focal spot of the femtosecond laser at a translation speed of 5-10 $\mu \mathrm{m} / \mathrm{s}$ in the porous glass immersed in water. The nanochannel is connected to a prefabricated microchannel through which the debris can be driven out from the nanochannel by bubble generation. Figure 19a presents a zoom-in view of the cross section of the nanochannel surrounded by small nanopores. The top view image gives a direct evidence on the formation of a single continuous nanochannel, as shown in Figure 19b. After the laser writing, the sample was annealed at a high temperature of $1120^{\circ} \mathrm{C}$ for $2 \mathrm{~h}$. Figure $19 \mathrm{c}$ shows a fluorescence microscope image of the postannealed nanochannels filled with fluorescent dyes, indicating that there are no clogs and leakage in the nanochannels.

Figure 18. (a-c) Evolution from nano-void array to single nano-void with decreasing laser intensity. The laser incident direction $(\mathbf{k})$, polarization $(\mathbf{E})$, and writing direction $(\mathbf{S})$ are indicated [37] (Reproduced with permission from OSA. C2013 by the Optical Society of America). (d-f) Principle schematic diagrams to achieve a single nanocrack [42] (Reproduced with permission from Springer. (C2014 by Springer).

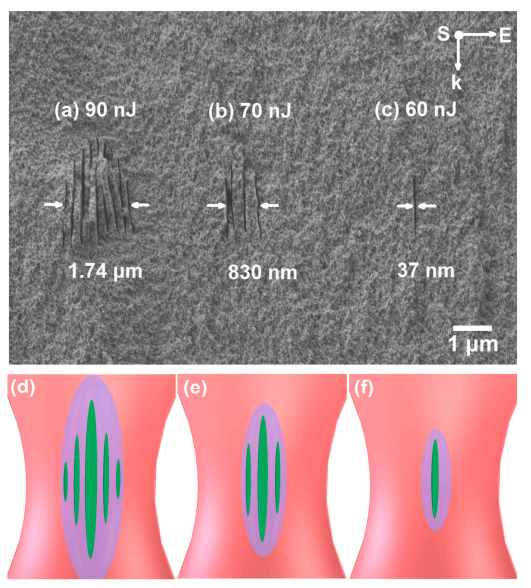


Figure 19. (a) Cross section view and (b) top view SEM images of a continuous nanocrack written in the porous glass. (c) Fluorescence microscope image of the postannealed nanofluidic channels filled with fluorescent dyes as an evidence that these nanochannels are through structures without clogging and leakage [37] (Reproduced with permission from OSA. (C)2013 by the Optical Society of America).

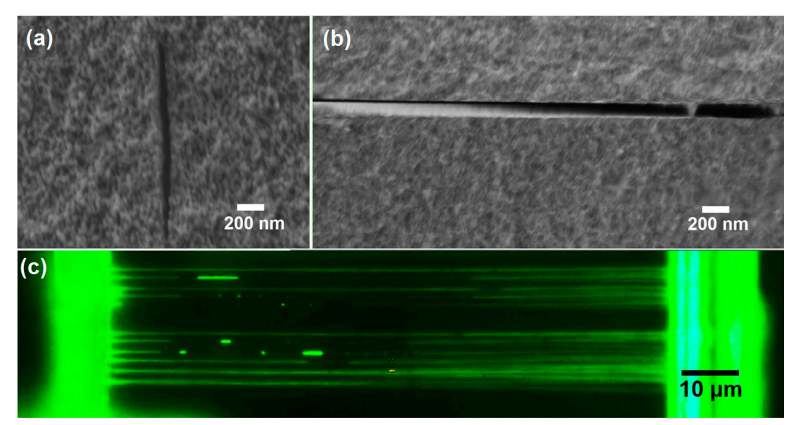

\subsection{Rapid Prototyping of 3D Nanofluidic Chip}

The unique 3D capability of femtosecond laser direct writing allows fabrication of nanochannels at arbitrary depths inside the glass substrate with a high axial resolution. Moreover, the dimensions of the channel can easily be controlled by adjusting the machining parameters, such as the laser pulse energy, the translation speed and the number of repeated scans. In combination with 3D microfluidics we have demonstrated in the previous sections, we now are capable of producing integrated micro-nanofluidic systems of complex 3D geometries and configurations in glass in a single continuous step.

Figure 20 shows a schematic diagram of the process flow chart for fabricating integrated 3D micro-nanofluidic structures by femtosecond laser direct writing in porous glass, showing four major steps: (1) fabrication of reservoirs by femtoseond laser direct write ablation of porous glass immersed in water with a low-NA objective (N.A. $=0.45$ ) at a pulse energy of $\sim 10 \mu \mathrm{J}$, as shown in Figure 20a; (2) direct writing of microchannels connecting the reservoirs with a long-working-distance water-immersed objective (N.A. $=1.10$ ) at a pulse energy of $0.5-3 \mu \mathrm{J}$, as shown in Figure 20b; (3) direct writing of a nanochannel array bridged between two microchannels with the same objective used in (2) but at a reduced pulse energy of 125-200 nJ, as shown in Figure 20c; and (4) postannealing of the glass substrate at $\sim 1120{ }^{\circ} \mathrm{C}$ for $120 \mathrm{~min}$, by which all the nanopores in porous glass can be collapsed to form a consolidated glass substrate, while the fabricated nanochannels could survive due to their larger diameter than the pores.

Figure 21a schematically illustrates an integrated micro-nanofluidic structure, which contains an array of two-layer nanochannels connected to two microfluidic channels. Figure $21 \mathrm{~b}$ shows the optical micrograph of the fabricated nanochannel arrays. The nanochannels were directly written at the depths of about $200 \mu \mathrm{m}$ below the porous glass surface, and the difference in the depths of the two layers of the nanochannels is only $\sim 1.5 \mu \mathrm{m}$. All the nanochannels were directly written by a single scan at a translating speed of $10 \mu \mathrm{m} / \mathrm{s}$ with a pulse energy of $128 \mathrm{~nJ}$. Note that as we have examined prior to the writing process, the threshold pulse energy for inducing optical breakdown in porous glass immersed in water is $\sim 108 \mathrm{~nJ}$. Therefore, the peak laser intensity we chose for writing the nanochannel is approximately $\sim 20 \%$ higher than the threshold intensity. The direction of laser polarization was perpendicular to the translating direction. During the fabrication of the nanochannel in porous glass immersed in water, it could be observed that the bubbles were continuously ejected from the inlet of 
the fabricated nanochannel into the thick microchannel, which can efficiently remove the debris produced by laser ablation. The continuous bubble flow in turn indicates that an unclogged nanochannel has been formed with a single scan (we observed previously that once clogging occurred, there would be no more bubbles coming out from the outlet.). After collapsing the nanopores by the postannealing, the size of each nanochannel was reduced by $\sim 14 \%$ isotropically in all three dimensions, resulting a total channel length of $\sim 40 \mu \mathrm{m}$, a width in the range of 30-50 nm, and a height in the range of $1-1.5 \mu \mathrm{m}$, as shown in Figure $21 \mathrm{~b}$,c. The cross section of the nanochannel appears highly elliptical, showing a typical depth-to-width ratio up to 25, as evidenced in Figure 21d. Such highly elliptical shape resembles the cross section of an individual nanoplane in a nanograting, indicating a relatively low fabrication resolution in the axial resolution.

Figure 20. Schematic diagram of process flow chart for fabricating 3D integrated micro-nanochannels by femtosecond laser direct writing.

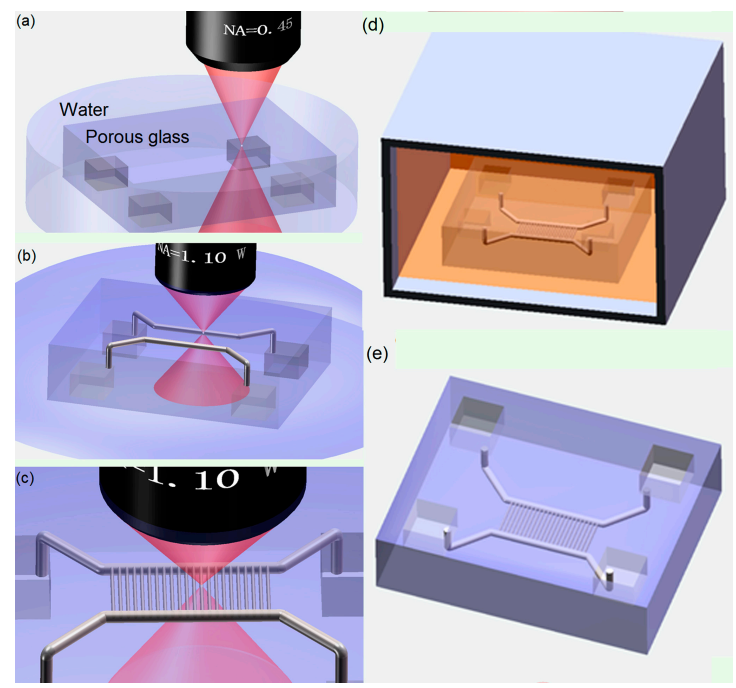

Figure 21. (a) Schematic diagram of an array of double-layer nanochannels to bridge two microchannels. (b) Top-view optical micrograph of double-layer nanochannels after postannealing. (c) Cross-sectional SEM micrograph of the nanochannels cleaved along the red dashed line in (b). The laser was incident from bottom of the figure, and the blue arrows indicate some tiny occasional protrusions on the edges of the nanochannels, which were formed due to the cleaving process. (d) Close-up SEM micrograph of cross section of a nanochannel [41] (Reproduced with permission from RSC. (C2013 by the Royal Society of Chemistry).

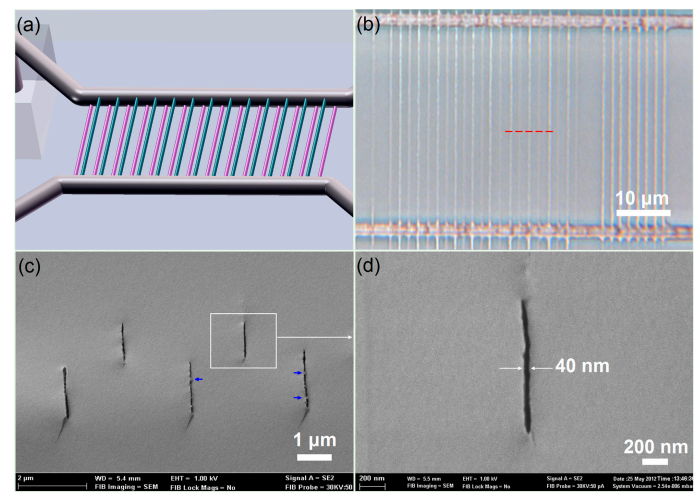


To demonstrate the applications of the fabricated nanochannels in investigating single molecular behaviors, we fabricate a complex 3D micro-nanofluidic device as illustrated in Figure 22a and perform an experiment of DNA stretching using the fabricated device. It is noteworthy that recently, nanochannel-based DNA stretching has attracted enormous attention because of its potential use in DNA analysis, whereas until now direct fabricating nanochannels and realizing 3D micro- and nanofluidic integration within bulk glass have not yet been achieved [81]. The device consists of two nanochannel arrays with different dimensions (i.e., widths and lengths) at different depths embedded in a single glass substrate. The nanochannels are connected to the four common reservoirs through two microchannels with a diameter of $\sim 50 \mu \mathrm{m}$. Such a device allows for simultaneously observing the stretching of DNA molecules in the two nanochannel arrays of different dimensions.

Figure $22 \mathrm{~b}$ presents a top view of the fabricated two nanochannel arrays, in which the short nanochannels have a length of $\sim 30 \mu \mathrm{m}$ and a cross section of $\sim 50 \mathrm{~nm} \times 1 \mu \mathrm{m}$, whereas the long nanochannles have a length of $\sim 100 \mu \mathrm{m}$ and a cross section of $\sim 200 \mathrm{~nm} \times 1.2 \mu \mathrm{m}$. After an aqueous buffer solution of stained $\lambda$ DNA was pipetted into a reservoir, the DNA molecules can readily transported into the nanochannels by capillary force. Figure $22 \mathrm{c}, \mathrm{d}$ is typical image of stretching of $\lambda$ DNA in the nanochannels. In the thin nanochannels with a cross section of $50 \mathrm{~nm} \times 1 \mu \mathrm{m}$, the average stretched length of DNA is $\sim 6.4 \pm 1.0 \mu \mathrm{m}$ ( $\sim 30 \%$ of the dye-adjusted contour length). In the thicker nanochannels with a cross section of $\sim 200 \mathrm{~nm} \times 1.2 \mu \mathrm{m}$, the average stretched length of DNA is $\sim 2.8 \pm 0.6 \mu \mathrm{m}(\sim 13 \%$ of the dye-adjusted contour length). The apparent lengths of some stretched DNA molecules in Figure $22 \mathrm{~d}$ can reach as long as $\sim 15 \mu \mathrm{m}$, which can be attributed to the clustering of the DNA molecules. As shown in these images, the continuous flow and uniform stretching of the DNA molecules in the nanochannel arrays also verify the continuity of the nanochannels and the repeatability of the direct writing process.

Figure 22. (a) Schematic diagram and (b) top-view optical micrograph of the fabricated 3D nanofluidic device for DNA analysis; and fluorescent images showing the stretching of $\lambda$ DNA in two arrays of nanochannels with different widths: (c) $50 \mathrm{~nm}$ and (d) $200 \mathrm{~nm}$ [41] (Reproduced with permission from RSC. (C2013 by the Royal Society of Chemistry).

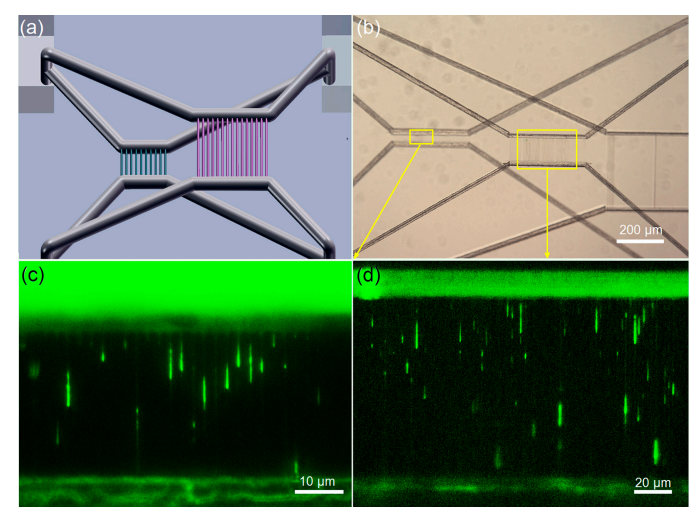

\section{Mechanism Investigation}

\subsection{Pulse Duration Dependence}

In previous section, we proposed that the mechanism behind the formation of a single nanochannel can be understood as a combination of the threshold effect and the formation of periodic nanograting 
during the process of laser writing in a porous glass immersed in water. Compared with traditional nanostructuring technique only utilizing threshold effect, the new-established nanostructuring strategy is relatively not sensitive to the laser peak intensity, and there exists a window (a range of laser intensity) for inducing a single nanochannel. Here, we show that the effective range of laser intensity for fabrication of nanochannel can be expanded by optimizing the laser pulse duration [42].

Figure 23 shows morphological changes of a single nanocrack induced by different laser powers and pulse durations. For the first row, the nanograting structures were written with $\sim 150$ fs pulses, whereas for the second and third row, the nanograting structures were written with $\sim 300 \mathrm{fs}$ pulses. One can see that when the laser intensity increases, the longitudinal length (i.e., the height) of the nanochannel increases, too, which is a manifestation of the threshold effect. The most significant information conveyed from these images is that at the $\sim 300$ fs pulse duration, the effective range of laser intensity for creating a single nanocrack has a broad range from $\sim 18 \mathrm{~mW}$ to $\sim 26 \mathrm{~mW}$, as shown in Figure $3 \mathrm{f}-\mathrm{o}$. In contrast, at the relatively shorter pulse duration of $\sim 150 \mathrm{fs}$, the effective range of laser intensity for creating a single nanocrack shrinks to a smaller range from $\sim 16 \mathrm{~mW}$ to $\sim 19 \mathrm{~mW}$, as shown in Figure 23a-e. In other word, in the case of nanograting fabrication with the $\sim 300$ fs pulses, the laser intensity can be varied by nearly $\sim 45 \%$ (i.e., (26-18)/18) while a single nanocrack structure can still be maintained. However, for the case of nanograting fabrication with the $\sim 150$ fs pulses, the variation of laser intensity has to be limited to $\sim 20 \%$ for avoiding the formation of double nanocracks.

Figure 23. SEM images of double and single nanocrack inside porous glass induced by different laser powers and pulse durations. (a-e) 150 fs; (f-o) 300 fs [42] (Reproduced with permission from Springer. (C2014 by Springer).

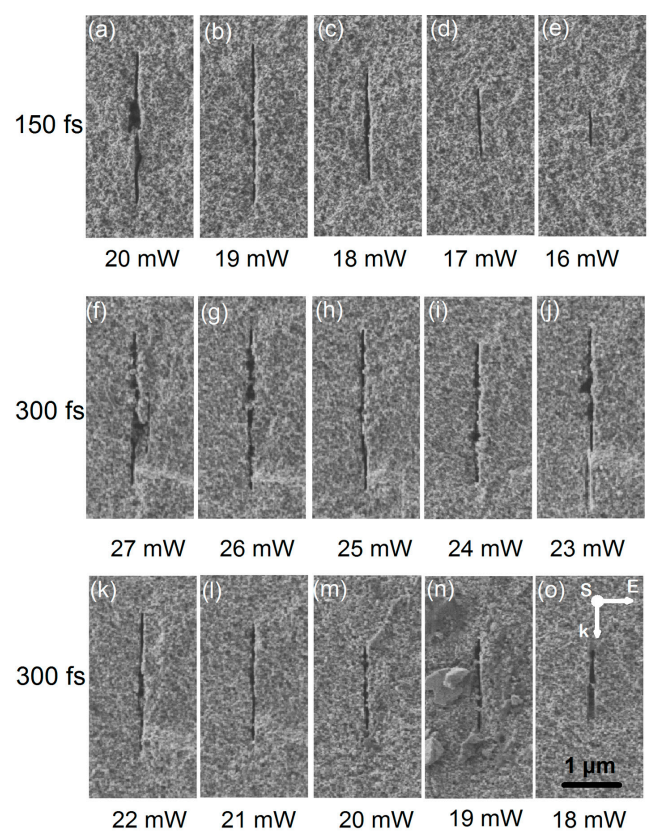

Figure 24 presents the pulse duration dependence of the threshold power for formation of a single nanocrack and double nanocracks. The range of laser intensity for producing only a single nanocrack in nanograting formation can be broadened by elongation of the laser pulse duration. However, when the pulse durations are longer than $\sim 300 \mathrm{fs}$, the roughness of sidewalls in the nanocracks becomes high probably due to the more severe heat effect. When the femtosecond laser pulses of a duration of 
$\sim 200$ fs are chosen, a sufficiently broad range of laser intensity ( $44 \%$ of the structuring threshold) for creating a single nanocrack can be achieved, while smooth sidewalls required by nanofluidics applications can still be maintained. The broadened processing window can make the fabrication more stable against the fluctuation of laser peak intensity and the fabricated nanofluidic structures more uniform. In addition, the dependence of the effective range of laser intensity for fabrication of nanochannel, which relies on the formation of a single nanocrack in a nanograting, will shed more light on the mechanism behind the nanograting formation in transparent materials with femtosecond laser irradiation.

Figure 24. The laser threshold power for formation of a single nanocrack and double nanocracks as functions of femtosecond laser pulse duration [42] (Reproduced with permission from Springer. (C2014 by Springer).

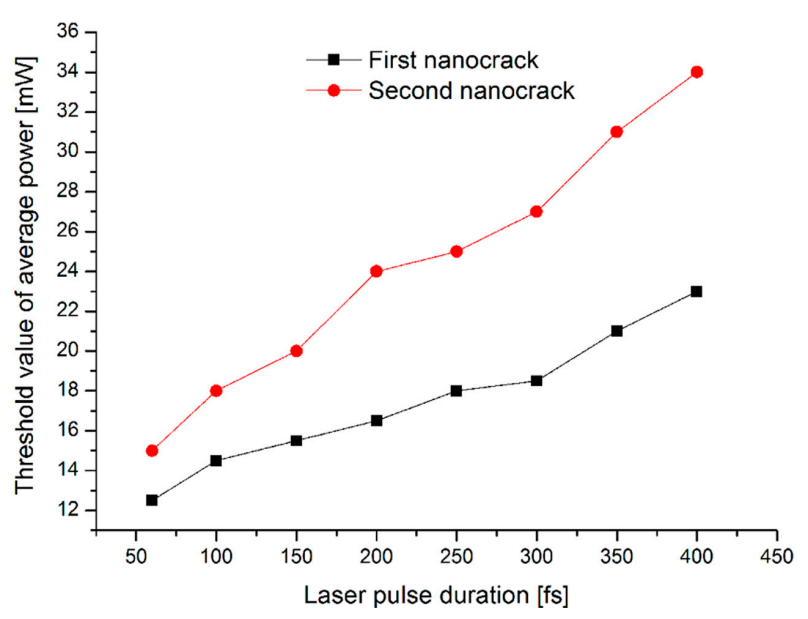

\subsection{Influence of Ionization Property of Immersion Liquid}

Over the recent decade, formation of nanogratings inside transparent materials by femtosecond laser irradiation has attracted broad attention because of its sub-diffraction-limit nature and its potential for optical, polarization sensitive and fluidic applications [82,83]. However, although great effort has been made on investigating the mechanism behind this phenomenon, a clear picture is still lacking until now $[75,84]$. Recently, it has been found that nanogratings can also be formed in a porous glass immersed in water by irradiation with femtosecond laser pulses of carefully selected pulse energies [37]. For the mechanism exploration, such porous material also provides unique opportunities to continuously tune the material properties by filling different types of solutions into the nanopores. For example, optical properties could be tuned by filling liquids with different nonlinear optical coefficients. Here, we place our focus on tuning the ionization property of the porous glass by doping the immersion water with $\mathrm{NaCl}$ at different concentrations [43].

Figure 25a-d shows the SEM images of a series of single nanocracks formed inside the porous glass immersed in water doped with $\mathrm{NaCl}$ at different concentrations. It can be concluded that the threshold power for inducing optical breakdown in the porous glass immersed in $\mathrm{NaCl}$ solution decreases with increasing of the concentration of $\mathrm{NaCl}$, while the width of a single nanocrack almost does not change. The same trend can also be seen in Figure 2e, in which the dependence of the threshold fluence of single-crack formation on the concentration of $\mathrm{NaCl}$ is plotted. 
Figure 26 quantitatively shows how the period of nanograting evolves with the increasing laser power and increasing concentration of $\mathrm{NaCl}$ in water. Generally speaking, at a fixed concentration of $\mathrm{NaCl}$, the period of nanogratings decreases with increasing power, which is consistent with the results reported previously $[85,86]$. On the other hand, we find that at a fixed output power of femtosecond laser, the period of nanogratings decreases with increasing concentration of $\mathrm{NaCl}$ in water. The comparison of these observations seems to suggest that a stronger ionization, which could be realized either by increasing the laser peak intensity or by reducing the ionization threshold, would lead to smaller period of the nanograting structures. This is consistent with the previous observation [87]. In our opinion, the decreasing period with enhancement of photoionization may suggest that an above critical density has been reached, resulting in a semiopaque plasma.

These results we obtained not only suggest a practical way for promoting the fabrication efficiency due to the reduction of the ablation threshold in water by doping of $\mathrm{NaCl}$, but also could shed new light on the mechanism behind the nanograting formation. Firstly, due to the fact that tuning of ionization threshold is achieved by changing the concentration of $\mathrm{NaCl}$ in water, as a result, it is the free electrons generated from the liquid that induce the subsequent ionization and ablation in the porous glass. Secondly, we may conclude that the formation of nanograting heavily depends on the density of electrons in the excitation region, as higher electron density could be achieved with either higher peak intensity or lower ionization potential, which in turn causes formation of the gratings of smaller period.

Figure 25. Nanogratings formed in porous glass by induced by femtosecond laser pulses at threshold fluencies in different immersion liquids: (a) water, (b) $0.01 \mathrm{M}$ of $\mathrm{NaCl}$ solution, (c) $0.06 \mathrm{M}$ of $\mathrm{NaCl}$ solution, and (d) $0.1 \mathrm{M}$ of the same solution. (e) Dependence of the threshold fluence of single-crack formation on the concentration of $\mathrm{NaCl}$ [43] (Reproduced with permission from OSA. (C2013 by the Optical Society of America).
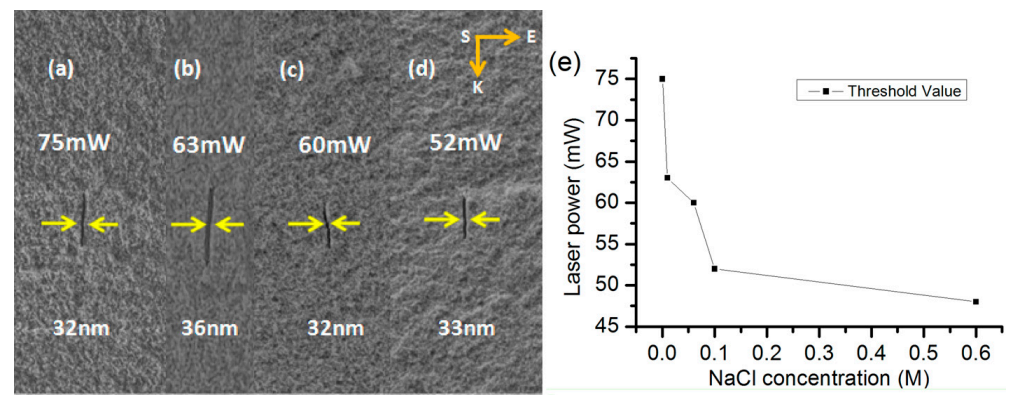

Figure 26. The dependence of nanograting period on laser power at different doping concentrations of $\mathrm{NaCl}$ [43] (Reproduced with permission from OSA. C2013 by the Optical Society of America).

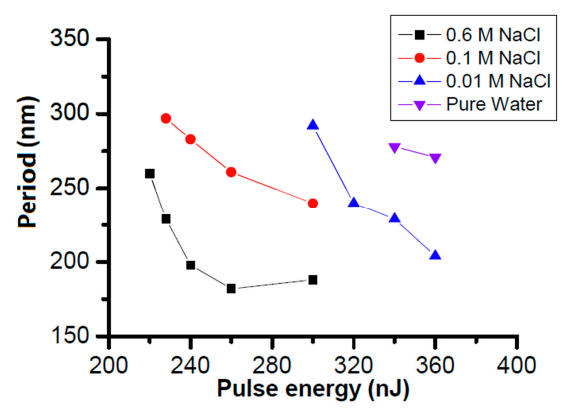




\section{Conclusions and Future Outlook}

In this review, we have shown that fabrication of 3D homogeneous microchannels with large sizes and complex geometries can be achieved using water-assisted femtosecond laser direct writing in porous glass followed by a postannelaing for consolidation of the porous glass. By further manipulating the laser pulse energy and polarization, nanofluidic channels with a width of $\sim 30 \mathrm{~nm}$, which is less than $\lambda / 25$ (here $\lambda=800 \mathrm{~nm}$, which is the wavelength of the writing beam), and an aspect ratio above $\sim 1000$ can be directly written in the porous glass, enabling straightforward integration of microfluidics and nanofluidics in a single platform. Interestingly, two extreme nonlinear effects, namely, the femtosecond-laser-induced nanograting formation and the threshold effect in femtosecond laser induced optical breakdown in glass, play important roles in fabrication of the nanochannels. Based on this technique, several functional devices built in glass that cannot be produced by any previous laser direct writing techniques, including a 3D passive microfluidic mixer, an integrated DNA analysis chip, etc., have been demonstrated. This technique will open a broad spectrum of opportunities for micro- and nanofluidic applications.

Currently, however, this emerging field is still in its infancy from both fundamental and applied points of views. First of all, the underlying physical mechanisms behind the formation of nanogratings as well as water-assisted femtosecond laser direct writing in porous glass are quite complicated and not yet fully understood. Secondly, the roughness of microfluidic structures fabricated by laser direct writing needs to be further improved for in vivo and optofluidic applications. Thirdly, the fabrication efficiencies for large-volume and high-aspect-ratio microfluidic devices are still quite low. At last, the cross section of the nanochannel appears highly elliptical and asymmetrical, indicating a relatively low fabrication resolution in the axial resolution. We expect that the future advance in 3D micro-/nano-fluidic applied research will strongly promote the further development and optimization of the technique.

\section{Acknowledgments}

This research is financially supported by National Natural Science Foundation of China (Nos. 11127901, 61275205, 61108015, 61221064 and 11104294). The authors would like to thank Dr. Ling Ling Qiao for assisting with preparation of the manuscript.

\section{Author Contributions}

Yang Liao has written the main manuscript, and Ya Cheng provided guidance and revised the manuscript.

\section{Conflicts of Interest}

The authors declare no conflict of interest.

\section{References}

1. Manz, A.; Graber, N.; Widmer, H.M. Miniaturized total chemical analysis systems: A novel concept for chemical sensing. Sens. Actuators B 1990, 1, 244-248. 
2. Whitesides, G.M. The origins and the future of microfluidics. Nature 2006, 442, 368-373.

3. Bellouard, Y.; Champion, A.; Lenssen, B.; Matteucci, M.; Schaap, A.; Beresna, M.; Corbari, C.; Gecevičius, M.; Kazansky, P.; Chappuis, O.; Kral, M.; Clavel, R.; Barrot, F.; Breguet, J.-M.; Mabillard, Y.; Bottinelli, S.; Hopper, M.; Hoenninger, C.; Mottay, E.; Lopez, J. The femtoprint project. J. Laser Micro/Nanoeng. 2012, 7, 1-10.

4. Xiong, W.; Zhou, Y.S.; He, X.N.; Gao, Y.; Samani, M.M.; Jiang, L.; Baldacchini, T.; Lu, Y.F. Simultaneous additive and subtractive three-dimensional micro/nano-fabrication using integrated two-photon polymerization and multi-photon ablation. Light Sci. Appl. 2012, 1, e6, doi:10.1038/1sa.2012.6.

5. Davis, K.M.; Miura, K.; Sugimoto, N.; Hirao, K. Writing waveguides in glass with a femtosecond laser. Opt. Lett. 1996, 21, 1729-1731.

6. Ams, M.; Dekker, P.; Marshall, G.D.; Withford, M.J. Monolithic $100 \mathrm{~mW} \mathrm{Yb}$ waveguide laser fabricated using the femtosecond-laser direct-write technique. Opt. Lett. 2009, 34, 247-249.

7. Chen, F.; Vazquez de Aldana, J.R. Optical waveguides in crystalline dielectric materials produced by femtosecond- laser micromachining. Laser Photon. Rev. 2014, 8, 251-275.

8. Marcinkevicius, A.; Juodkazis, S.; Watanabe, M.; Miwa, M.; Matsuo, S.; Misawa, H.; Nishii, J. Femtosecond laser-assisted three-dimensional microfabrication in silica. Opt. Lett. 2001, 26, 277-279.

9. Masuda, M.; Sugioka, K.; Cheng, Y.; Aoki, N.; Kawachi, M.; Shihoyama, K.; Toyoda, K.; Helvajian, H.; Midorikawa, K. 3-D microstructuring inside photosensitive glass by femtosecond laser excitation. Appl. Phys. A 2003, 76, 857-860.

10. Matsuo, S.; Kiyama, S.; Shichijo, Y.; Tomita, T.; Hashimoto, S.; Hosokawa, Y.; Masuhara, H. Laser microfabrication and rotation of ship-in-a-bottle optical rotators. Appl. Phys. Lett. 2008, 93, 051107.

11. Masuda, M.; Sugioka, K.; Cheng, Y.; Hongo, T.; Shihoyama, K.; Takai, H.; Miyaloto, I.; Midorikawa, K. Direct fabrication of freely movable microplate inside photosensitive glass by femtosecond laser for lab-on-chip application. Appl. Phys. A 2004, 78, 1029-1032.

12. Cheng, Y.; Sugioka, K.; Midorikawa, K.; Masuda, M.; Toyoda, K.; Kawachi, M.; Shihoyama, K. Three-dimensional micro-optical components embedded in photosensitive glass by a femtosecond laser. Opt. Lett. 2003, 28, 1144-1146.

13. Cheng, Y.; Tsai, H.L.; Sugioka, K.; Midorikawa, K. Fabrication of 3D microoptical lenses in photosensitive glass using femtosecond laser micromachining. Appl. Phys. A 2006, 85, 11-14.

14. Wang, Z.; Sugioka, K.; Midorikawa, K. Fabrication of integrated microchip for optical sensing by femtosecond laser direct writing of Foturan glass. Appl. Phys. A 2007, 89, 951-955.

15. Cheng, Y.; Sugioka, K.; Midorikawa, K. Microfluidic laser embedded in glass by three-dimensional femtosecond laser microprocessing. Opt. Lett. 2004, 29, 2007-2009.

16. Hanada, Y.; Sugioka, K.; Kawano, H.; Ishikawa, I.S.; Miyawaki, A.; Midorikawa, K. Nano-aquarium for dynamic observation of living cells fabricated by femtosecond laser direct writing of photostructurable glass. Biomed. Microdevices 2008, 10, 403-410.

17. Hanada, Y.; Sugioka, K.; Ishikawa, I.S.; Kawano, H.; Miyawaki, A.; Midorikawa, K. 3D microfluidic chips with integrated functional microelements fabricated by a femtosecond laser for studying the gliding mechanism of cyanobacteria. Lab Chip 2011, 11, 2109-2115. 
18. Crespi, A.; Gu, Y.; Ngamsom, B.; Hoekstra, H.J.W.M.; Dongre, C.; Pollnau, M.; Ramponi, R.; van den Vlekkert, H.H.; Watts, P.; Cerullo, G.; Osellame, R. Three-dimensional Mach-Zehnder interferometer in a microfluidic chip for spatially-resolved label-free detection. Lab. Chip 2010, 10, 1167-1173.

19. Kim, M.; Hwang, D.J.; Jeon, H.; Hiromatsu, K.; Grigoropoulos, C.P. Single cell detection using a glass-based optofluidic device fabricated by femtosecond laser pulses. Lab. Chip 2009, 9, 311-318.

20. Bragheri, F.; Ferrara, L.; Bellini, N.; Vishnubhatla, K.C.; Minzionil, P.; Ramponi, R.; Osellame, R.; Cristiani, I. Optofluidic chip for single cell trapping and stretching fabricated by a femtosecond laser. J. Biophoton. 2010, 3, 234-243.

21. Schaap, A.; Bellouard, Y.; Rohrlack, T. Optofluidic lab-on-a-chip for rapid algae population screening. Biomed. Opt. Express 2011, 2, 658-664.

22. Xu, J.; Liao, Y.; Zeng, H.D.; Sun, H.Y.; Song, J.; Wang, X.S.; Cheng, Y.; Xu, Z.Z.; Sugioka, K.; Midorikawa, K. Selective metallization on insulator surfaces with femtosecond laser pulses. Opt. Express 2007, 15, 12743-12748.

23. Liao, Y.; Xu, J.; Sun, H.Y.; Song, J.; Wang, X.S.; Cheng, Y. Fabrication of microelectrodes deeply embedded in $\mathrm{LiNbO}_{3}$ using a femtosecond laser. Appl. Surf. Sci. 2008, 254, 7018-7021.

24. Liao, Y.; Xu, J.; Cheng, Y.; Zhou, Z.H.; He, F.; Sun, H.Y.; Song, J.; Wang, X.S.; Xu, Z.Z.; Sugioka, K.; Midorikawa, K. Electro-optic integration of embedded electrodes and waveguides in $\mathrm{LiNbO}_{3}$ using a femtosecond laser. Opt. Lett. 2008, 33, 2281-2283.

25. Xu, J.; Wu, D.; Hanada, Y.; Chen, C.; Wu, S.Z.; Cheng, Y.; Sugioka, K.; Midorikawa, K. Electrofluidics fabricated by space-selective metallization in glass microfluidic structures using femtosecond laser direct writing. Lab Chip 2013, 13, 4608-4616.

26. Liao, Y.; Qiao, L.; Wang, Z.; Wang, M.; Liu, L.; Sugioka, K.; Cheng, Y. Fabrication of a liquid crystal light modulator by use of femtosecond-laser-induced nanoripples. Opt. Mater. Express 2013, 3, 1698-1704.

27. Bellouard, Y.; Said, A.; Bado, P. Integrating optics and micro-mechanics in a single substrate: A step toward monolithic integration in fused silica. Opt. Express 2005, 13, 6635-6644.

28. Lenssen, B.; Bellouard, Y. Optically transparent glass micro-actuator fabricated by femtosecond laser exposure and chemical etching. Appl. Phys. Lett. 2012, 101, 103503.

29. Hnatovsky, C.; Taylor, R.S.; Simova, E.; Rajeev, P.P.; Rayner, D.M.; Bhardwaj, V.R.; Corkum, P.B. Fabrication of microchannels in glass using focused femtosecond laser radiation and selective chemical etching. Appl. Phys. A 2006, 84, 47-61.

30. Sugioka, K.; Cheng, Y.; Midorikawa, K. Three-dimensional micromachining of glass using femtosecond laser for lab-on-a-chip device manufacture. Appl. Phys. A 2005, 81, 1-10.

31. Sugioka, K.; Hanada, Y.; Midorikawa, K. Three-dimensional femtosecond laser micromachining of photosensitive glass for biomicrochips. Laser Photonics Rev. 2010, 4, 386-400.

32. Li, Y.; Itoh, K.; Watanabe, W.; Yamada, K.; Kuroda, D.; Nishii, J.; Jiang, Y.Y. Three-dimensional hole drilling of silica glass from the rear surface with femtosecond laser pulses. Opt. Lett. 2001, 26, 1912-1914.

33. Maruo, S.; Fourkas, J.T. Recent progress in multiphoton microfabrication. Laser Photon. Rev. 2008, 2, 100-111. 
34. Ke, K.; Hasselbrink, E.F.; Hunt, A.J. Rapidly prototyped three-dimensional nanofluidic channel networks in glass substrates. Anal. Chem. 2005, 77, 5083-5088.

35. Liao, Y.; Ju, Y.; Zhang, L.; He, F.; Zhang, Q.; Shen, Y.; Chen, D.; Cheng, Y.; Xu, Z.; Sugioka, K.; Midorikawa, K. Three-dimensional microfluidic channel with arbitrary length and configuration fabricated inside glass by femtosecond laser direct writing. Opt. Lett. 2010, 35, 3225-3227.

36. Liu, C.N.; Liao, Y.; He, F.; Lin, D.; Song, J.X.; Lin, J.T.; Cheng, Y.; Sugioka, K.; Midorikawa, K. Compact 3D microfluidic channel structures embedded in glass fabricated by femtosecond laser direct writing. J. Laser Micro/Nanoeng. 2013, 8, 170-174.

37. Liao, Y.; Shen, Y.L.; Qiao, L.L.; Chen, D.P.; Cheng, Y.; Sugioka, K.; Midorikawa, K. Femtosecond laser nanostructuring in porous glass with sub-50nm feature sizes. Opt. Lett. 2013, $38,187-189$.

38. Ju, Y.; Liao, Y.; Zhang, L.; Zhang, Q.; Shen, Y.; Chen, D.; Cheng, Y.; Xu, Z.; Sugioka, K.; Midorikawa, K. Fabrication of large-volume microfluidic chamber embedded in glass using three-dimensional femtosecond laser micromachining. Microfluid. Nanofluid. 2011, 11, 111-117.

39. Liao, Y.; Song, J.; Li, E.; Luo, Y.; Shen, Y.; Chen, D.; Cheng, Y.; Xu, Z.; Sugioka, K.; Midorikawa, K. Rapid prototyping of three-dimensional microfluidic mixers in glass by femtosecond laser direct writing. Lab Chip 2012, 12, 746-749.

40. Ju, Y.; Liu, C.; Liao, Y.; Liu, Y.; Zhang, L.; Shen, Y.; Chen, D.; Cheng, Y. Direct fabrication of a microfluidic chip for electrophoresis analysis by water-assisted femtosecond laser writing in porous glass. Chin. Opt. Lett. 2013, 11, 072201.

41. Liao, Y.; Cheng, Y.; Liu, C.N.; Song, J.X.; He, F.; Shen, Y.L.; Chen, D.P.; Xu, Z.Z.; Fan, Z.C.; Wei, X.B.; Sugioka, K.; Midorikawa, K. Direct laser writing of sub-50nm nanofluidic channels buried in glass for threedimensional micro-nanofluidic integration. Lab Chip 2013, 13, 1626-1631.

42. Liao, Y.; Zeng, B.; Qiao, L.L.; Liu, L.Q.; Sugioka, K.; Cheng, Y. Threshold effect in femtosecond laser induced nanograting formation in glass: influence of the pulse duration. Appl. Phys. A 2014, $114,223-230$.

43. Umran, F.A.; Liao, Y.; Elias, M.M.; Sugioka, K.; Stoian, R.; Cheng, G.H.; Cheng, Y. Formation of nanogratings in a transparent material with tunable ionization property by femtosecond laser irradiation. Opt. Express 2013, 21, 15259-15267.

44. Elmer, T.H. Porous and Reconstructed Glasses. In Engineered Materials Handbook; ASM International: Metals Park, OH, USA, 1992; Volume 4, pp. 427-432.

45. Song, J.; Wang, X.; Hu, X.; Dai, Y.; Qiu, J.; Cheng, Y.; Xu, Z. Formation mechanism of self-organized voids in dielectrics induced by tightly focused femtosecond laser pulses. Appl. Phys. Lett. 2008, 92, 092904.

46. Hwang, D.J.; Choi, T.Y.; Grigoropoulos, C.P. Liquid-assisted femtosecond laser drilling of straight and three-dimensional microchannels in glass. Appl. Phys. A 2004, 79, 605-612.

47. Herman, P.R.; Oettl, A.; Chen, K.P.; Marjoribanks, R.S. Laser micromachining of transparent fused silica with 1-ps pulses and pulse trains. Proc. SPIE 1999, 3616, 148-155.

48. Anderson, J.R.; Chiu, D.T.; Jackman, R.J.; Cherniavskaya, O.; McDonald, J.C.; Wu, H.; Whitesides, S.H.; Whitesides, G.M. Fabrication of topologically complex three-dimensional microfluidic systems in PDMS by rapid prototyping. Anal. Chem. 2000, 72, 3158-3164. 
49. Zhu, J.J.; Xuan, X.C. Particle electrophoresis and dielectrophoresis in curved microchannels. J. Colloid Interface Sci. 2009, 340, 285-290.

50. Garcia, A.L.; Ista, L.K.; Petsev, D.N.; O’Brien, M.J.; Bisong, P.; Mammoli, A.A.; Brueck, S.R. J.; Lopez, G.P. Electrokinetic molecular separation in nanoscale fluidic channels. Lab Chip 2005, 11, 1271-1276.

51. Liu, C.N.; Liao, Y.; He, F.; Shen, Y.L.; Chen, D.P.; Cheng, Y.; Xu, Z.Z.; Sugioka, K.; Midorikawa, K. Fabrication of three-dimensional microfluidic channels inside glass using nanosecond laser direct writing. Opt. Express 2012, 20, 4291-4296.

52. Wang, J.; Niino, H.; Yabe, A. One-step microfabrication of fused silica by laser ablation of an organic solution. Appl. Phys. A 1999, 68, 111-113.

53. Niino, H.; Yasui, Y.; Ding, X.; Narazaki, A.; Sato, T.; Kawaguchi, Y.; Yabe, A. Surface micro-fabrication of silica glass by excimer laser irradiation of organic solvent. J. Photochem. Photo. A Chem. 2003, 158, 179-182.

54. Vass, C.; Osvay, K.; Hopp, B. Fabrication of $150 \mathrm{~nm}$ period grating in fused silica by two-beam interferometric laser induced backside wet etching method. Opt. Express 2006, 14, 8354-8359.

55. Abbott, A. Cell culture: Biology's new dimension. Nature 2003, 424, 870-872.

56. Nguyen, N.T.; Wu, Z.G. Micromixers-A review. J. Micromech. Microeng. 2005, 15, R1, doi:10.1088/0960-1317/15/2/R01.

57. Liu, R.H.; Stremler, M.A.; Sharp, K.V.; Olsen, M.G.; Santiago, J.G.; Adrian, R.J.; Aref, H.; Beebe, D.J. Passive mixing in a three-dimensional serpentine microchannel. J. Microelectromech. Syst. 2000, 9, 190-197.

58. Stroock, A.D.; Dertinger, S.K.; Ajdari, A.; Mezic, I.; Stone, H.A.; Whitesides, G.M. Chaotic mixer for microchannels. Science 2002, 295, 647-651.

59. Therriault, D.; White, S.R.; Lewis, J.A. Chaotic mixing in three-dimensional microvascular networks fabricated by direct-write assembly. Nat. Mater. 2003, 2, 265-271.

60. Chen, H.; Meiners, J.C. Topologic mixing on a microfluidic chip. Appl. Phys. Lett. 2004, 84, 2193-2195.

61. Schönfeld, F.; Hessel, V.; Hofmann, C. An optimised split-and-recombine micro-mixer with uniform "chaotic" mixing. Lab Chip 2004, 4, 65-69.

62. Park, S.J.; Kim, J.K.; Park, J.; Chung, S.; Chung, C.; Chang, J.K. Rapid three-dimensional passive rotation micromixer using the breakup process. J. Micromech. Microeng. 2004, 14, 6-14.

63. Xia, H.M.; Wan, S.Y. M.; Shu, C.; Chew, Y.T. Chaotic micromixers using two-layer crossing channels to exhibit fast mixing at low Reynolds numbers. Lab Chip 2005, 5, 748-755.

64. Yasui, T.; Omoto, Y.; Osato, K.; Kaji, N.; Suzuki, N.; Naito, T.; Watanabe, M.; Okamoto, Y.; Tokeshi, M.; Shamoto, E.; Baba, Y. Microfluidic baker's transformation device for three-dimensional rapid mixing. Lab Chip 2011, 11, 3356-3360.

65. Wiggins, S.; Ottino, J.M. Foundations of chaotic mixing. Philos. Trans. R. Soc. London Ser. A 2004, 362, 937-970.

66. Carrière, P. On a three-dimensional implementation of the baker's transformation. Phys. Fluids 2007, 19, 118110.

67. Sugioka, K.; Cheng, Y. Femtosecond laser processing for optofluidic fabrication. Lab Chip 2012, 12, 3576-3589. 
68. Dongre, C.; van Weerd, J.; Bellini, N.; Osellame, R.; Cerullo, G.; van Weeghel, R.; Hoekstra, H.J.W.M.; Pollnau, M. Dual-point dual-wavelength fluorescence monitoring of DNA separation in a lab on a chip. Biomed. Opt. Express 2010, 1, 729-735.

69. Abgrall, P.; Nguyen, N.T. Nanofluidic devices and their applications. Anal. Chem. 2008, 80, 2326-2341.

70. Kovarik, M.L.; Jacobson, S.C. Nanofluidics in Lab-on-a-Chip Devices. Anal. Chem. 2009, 81, 7133-7140.

71. Kuo, T.C.; Cannon, D.M.; Shannon, M.A.; Bohn, P.W.; Sweedler, J.V. Hybrid threedimensional nanofluidic/microfluidic devices using molecular gates. Sens. Actuators A 2003, 102, 223-233.

72. Jeon, S.; Malyarchuk, V.; White, J.O.; Rogers, J.A. Optically fabricated three dimensional nanofluidic mixers for microfluidic devices. Nano Lett. 2005, 5, 1351-1356.

73. Lee, S.; An, R.; Hunt, A.J. Liquid glass electrodes for nanofluidics. Nat. Nanotechnol. 2010, 5 , 412-416.

74. Shimotsuma, Y.; Kazansky, P.; Qiu, J.; Hirao, K. Self-organized nanogratings in glass irradiated by ultrashort light pulses. Phys. Rev. Lett. 2003, 91, 247405.

75. Bhardwaj, V.R.; Simova, E.; Rajeev, P.P.; Hnatovsky, C.; Taylor, R.S.; Rayner, D.M.; Corkum, P.B. Optically produced arrays of planar nanostructures inside fused silica. Phys. Rev. Lett. 2006, 96, 057404.

76. Hnatovsky, C.; Taylor, R.S.; Simova, E.; Bhardwaj, V.R.; Rayner, D.M.; Corkum, P.B. Polarization-selective etching in femtosecond laser-assisted microfluidic channel fabrication in fused silica. Opt. Lett. 2005, 30, 1867-1869.

77. Korte, F.; Serbin, J.; Koch, J.; Egbert, A.; Fallnich, C.; Ostendorf, A.; Chichkov, B.N. Towards nanostructuring with femtosecond laser pulses. Appl. Phys. A 2003, 77, 229-235.

78. Joglekar, A.P.; Liu, H.; Meyhofer, E.; Mourou, G.; Hunt, A.J. Optics at critical intensity: Applications to nanomorphing. Proc. Natl. Acad. Sci. USA 2004, 101, 5856-5861.

79. Fischer, J.; Wegener, M. Three-dimensional optical laser lithography beyond the diffraction limit. Laser Photonics Rev. 2013, 7, 22-44.

80. Juodkazis, S.; Nishimura, K.; Tanaka, S.; Misawa, H.; Gamaly, E.G.; Luther-Davies, B.; Hallo, L.; Nicolai, P.; Tikhonchuk, V.T. Laser-induced microexplosion confined in the bulk of a sapphire crystal: evidence of multimegabar pressures. Phys. Rev. Lett. 2006, 96, 166101.

81. Tegenfeldt, J.O.; Prinz, C.; Cao, H.; Huang, R.L.; Austin, R.H.; Chou, S.Y.; Cox, E.C.; Sturm, J.C. Micro- and nanofluidics for DNA analysis. Anal. Bioanal. Chem. 2004, 378, 1678-1692.

82. Taylor, R.; Hnatovsky, C.; Simova, E. Applications of femtosecond laser induced self-organized planar nanocracks inside fused silica glass. Laser Photon. Rev. 2008, 2, 26-46.

83. Cheng, G.; Mishchik, K.; Mauclair, C.; Audouard, E.; Stoian, R. Ultrafast laser photoinscription of polarization sensitive devices in bulk silica glass. Opt. Express 2009, 17, 9515-9525.

84. Richter, S.; Heinrich, M.; Doring, S.; Tünnermann, A.; Nolte, S.; Peschel, U. Nanogratings in fused silica: Formation, control, and applications. J. Laser Appl. 2012, 24, 042008.

85. Richter, S.; Heinrich, M.; Doring, S.; Tuennermann, A.; Nolte, S. Formation of femtosecond laser-induced nanogratings at high repetition rates. Appl. Phys. A 2011, 104, 503-507. 
86. Mauclair, C.; Zamfirescu, M.; Colombier, J.P.; Cheng, G.; Mishchik, K.; Audouard, E.; Stoian, R. Control of ultrafast laser-induced bulk nanogratings in fused silica via pulse time envelopes. Opt. Express 2012, 20, 12997-13005.

87. Kazansky, P.G.; Shimotsuma, Y. Self-assembled sub-wavelength structures and form birefringence created by femtosecond laser writing in glass: properties and applications. J. Ceram. Soc. Jpn. 2008, 116, 1052-1062.

(C) 2014 by the authors; licensee MDPI, Basel, Switzerland. This article is an open access article distributed under the terms and conditions of the Creative Commons Attribution license (http://creativecommons.org/licenses/by/4.0/). 